\title{
Intramolecular Diels-Alder Reactions of Siloxacyclopentene Constrained Trienes
}

\author{
Geoff T. Halvorsen and William R. Roush* \\ Departments of Chemistry and Biochemistry \\ Scripps Florida, Jupiter, FL 33458
}

e-mail: roush@scripps.edu

Supporting Information:

Experimental Procedures for Synthesis and Diels-Alder Reactions of

Trienes 7a-7e and 10a-10e

SI-1 
General Experimental Details: All reaction solvents were purified before use. THF, $\mathrm{CH}_{2} \mathrm{Cl}_{2}$, and toluene were purified by passing through a solvent column composed of activated $\mathrm{A}-1$ alumina. $\mathrm{NEt}_{3}$ was distilled from calcium hydride prior to use. Acrolein was distilled prior to use and stored with $1 \% \mathrm{w} / \mathrm{w}$ of hydroquinone as a stabilizing agent. Acrolein pinacol acetal was prepared according to literature procedure. Unless otherwise indicated all other chemicals were used as purchased without further purification.

Physical Properties and Spectroscopic Measurements: Proton nuclear magnetic resonance ( $\left({ }^{1} \mathrm{H}\right.$ NMR) spectra and carbon-13 $\left({ }^{13} \mathrm{C}\right)$ NMR spectra were recorded at $400 \mathrm{MHz}$ and $100 \mathrm{MHz}$ respectively. The proton signal of residual, non-dueterated solvent $(\delta 7.26 \mathrm{ppm}$ for $\mathrm{CHCl}_{3}$ ) was used as a reference for ${ }^{1} \mathrm{H}$ spectra. For ${ }^{13} \mathrm{C}$ spectra, chemical shifts are reported relative to the $\delta 77.0 \mathrm{ppm}$ resonance of $\mathrm{CDCl}_{3}$. Coupling constants are reported in $\mathrm{Hz}$. Infrared spectra (IR) were recorded as thin films on an FTIR instrument. Mass spectra were recorded at the University of Michigan Mass Spectrometry Laboratory.

Analytical thin layer chromatography (TLC) was performed on Kieselgel $60 \mathrm{~F}_{254}$ glass plates precoated with $0.25 \mathrm{~mm}$ thickness of silica gel. The TLC plates were visualized under UV light and/or by staining with ceric ammonium molybdate $\left(5 \mathrm{~g} \mathrm{Ce}\left(\mathrm{SO}_{4}\right)_{2}, 25 \mathrm{~g}\right.$ $\left(\mathrm{NH}_{4}\right) \mathrm{Mo}_{7} \mathrm{O}_{24} \bullet 4 \mathrm{H}_{2} \mathrm{O}, 450 \mathrm{~mL} \mathrm{H} \mathrm{H}_{2} \mathrm{O}, 50 \mathrm{~mL} \mathrm{H} \mathrm{SO}_{4}$ ). Column chromatography was performed using Kieselgel 60 (230-400 mesh) silica gel. 


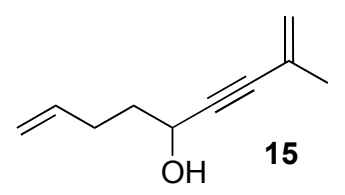

2-MethyInona-1,8-dien-3-yn-5-ol (15). To a solution of oxalyl chloride $(0.95 \mathrm{~mL}, 11$ mmol) in $\mathrm{CH}_{2} \mathrm{Cl}_{2}(25 \mathrm{~mL})$ at $-70{ }^{\circ} \mathrm{C}$ was added dimethylsulfoxide $(1.7 \mathrm{~mL}, 24 \mathrm{mmol})$ in $\mathrm{CH}_{2} \mathrm{Cl}_{2}$ (5 mL), followed by 5-penten-1-ol (13) $(1 \mathrm{~mL}, 10 \mathrm{mmol})$ in $\mathrm{CH}_{2} \mathrm{Cl}_{2}(10 \mathrm{~mL})$. The mixture was stirred for $5 \mathrm{~min}$, then $\mathrm{NEt}_{3}(7 \mathrm{~mL}, 50 \mathrm{mmol})$ was added dropwise. The solution was stirred at $-78 \mathrm{C}$ for $10 \mathrm{~min}$ then warmed to room temperature. The mixture was then diluted with $\mathrm{CH}_{2} \mathrm{Cl}_{2}$ and quenched with water. The aqueous layer was extracted with $\mathrm{CH}_{2} \mathrm{Cl}_{2}$, and the combined organic layers were washed twice with $1 \mathrm{M} \mathrm{HCl}$ (aq.) then brine. The organic layer was then dried over anhydrous $\mathrm{Na}_{2} \mathrm{SO}_{4}$ and concentrated in vacuo. The resulting oil was used without further purification.

To a solution of 2-methylbut-1-en-3-yne $(1 \mathrm{~mL}, 11.5 \mathrm{mmol})$ in THF $(10 \mathrm{~mL})$ at $0^{\circ} \mathrm{C}$ was added $n$-BuLi $(4.45 \mathrm{~mL}, 11 \mathrm{mmol}, 2.5 \mathrm{M}$ in hexanes). This solution was stirred for $15 \mathrm{~min}$ followed by addition of the crude aldehyde from the preceding step. The solution was allowed to warm to room temperature slowly over $1.5 \mathrm{~h}$ at which time it was diluted with $\mathrm{Et}_{2} \mathrm{O}$ and quenched with saturated aqueous ammonium chloride. The aqueous layer was extracted with $\mathrm{Et}_{2} \mathrm{O}$, then the combined organic layers were washed with brine, dried over anhydrous $\mathrm{Na}_{2} \mathrm{SO}_{4}$ and concentrated in vacuo. The resulting orange oil was purified by column chromatography $\left(9: 1\right.$ hexanes/ $\left./ \mathrm{Et}_{2} \mathrm{O}\right)$ yielding $0.98 \mathrm{~g}(66 \%)$ of 11 as a pale yellow oil: ${ }^{1} \mathrm{HNMR}\left(\mathrm{CDCl}_{3}, 400 \mathrm{MHz}\right) \delta 5.85$ (ddt, J = 17.2, , 6.7Hz, 1H), $5.29(\mathrm{~s}, 1 \mathrm{H}), 5.23(\mathrm{~s}, 1 \mathrm{H}), 5.08$ $(\mathrm{d}, \mathrm{J}=17.2 \mathrm{~Hz}, 1 \mathrm{H}), 5.00(\mathrm{~d}, \mathrm{~J}=10.3, \mathrm{~Hz}, 1 \mathrm{H}), 4.51(\mathrm{q}, \mathrm{J}=6.1 \mathrm{~Hz}, 1 \mathrm{H}), 2.24(\mathrm{q}, \mathrm{J}=7.3 \mathrm{~Hz}$, $2 \mathrm{H}), 1.89(\mathrm{~s}, 3 \mathrm{H}), 1.87-1.77(\mathrm{~m}, 3 \mathrm{H}) ;{ }^{13} \mathrm{CNMR}\left(\mathrm{CDCl}_{3}, 100 \mathrm{MHz}\right) \delta 137.7,126.3,122.2$, 115.3, 88.8, 86.3, 62.3, 36.9, 29.4, 23.4; FTIR (Thin film) $v$ max 3334 (br), 3097, 3079, 2977 , 2948, 2923, 2860, 2224, 1809, 1641, 1614, 1419, 1374, 1336, 1287, 1123, 1051, 1013, 910 , 792, 774, 726, $643 \mathrm{~cm}^{-1}$; HRMS (ESI) calcd for $\mathrm{C}_{10} \mathrm{H}_{14} \mathrm{O}[\mathrm{M}+\mathrm{Na}]^{+} 173.0942 \mathrm{~m} / \mathrm{z}$ found $173.0948 \mathrm{~m} / \mathrm{z}$.

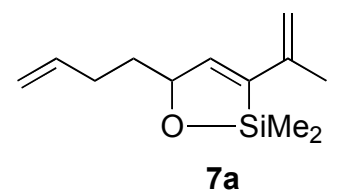

5-(But-3-enyl)-2,2-dimethyl-3-(prop-1-en-2-yl)-2,5-dihydro-1,2-oxasilole (7a). To $15(4.43 \mathrm{~g}, 29.5 \mathrm{mmol})$ was added tetramethyldisilazane $(3.1 \mathrm{~mL}, 18 \mathrm{mmol})$. The solution 
was stirred for $12 \mathrm{~h}$ then the volatile material was removed in vacuo. The resulting yellow oil was dissolved in THF $(100 \mathrm{~mL})$, and a solution of KOt-Bu $(330 \mathrm{mg}, 3 \mathrm{mmol})$ in THF $(15 \mathrm{~mL})$ was added dropwise. The solution was stirred for 1 hour, then diluted with $\mathrm{Et}_{2} \mathrm{O}$ and quenched with saturated aqueous ammonium chloride. The aqueous layer was extracted with $\mathrm{Et}_{2} \mathrm{O}$. The combined organic layers were then washed with brine, dried over anhydrous $\mathrm{Na}_{2} \mathrm{SO}_{4}$ and concentrated in vacuo. The crude product was purified by column chromatography (1\% $\mathrm{NEt}_{3}$ in hexanes) to yield $5.18 \mathrm{~g}(85 \%)$ of $7 \mathrm{a}$ as a pale yellow oil: ${ }^{1} \mathrm{HNMR}\left(\mathrm{CDCl}_{3}, 400 \mathrm{MHz}\right) \delta 6.52(\mathrm{~d}, \mathrm{~J}=1.8 \mathrm{~Hz}, 1 \mathrm{H}) 5.85$ (ddt, J = 17.1, 10.2, $\left.6.6 \mathrm{~Hz}, 1 \mathrm{H}\right)$, $5.04(\mathrm{dq}, \mathrm{J}=17.1,1.7 \mathrm{~Hz}, 1 \mathrm{H}), 5.00(\mathrm{~s}, 1 \mathrm{H}), 4.96(\mathrm{ddt}, \mathrm{J}=10.2,2,1.3 \mathrm{~Hz}, 1 \mathrm{H}), 4.83(\mathrm{~s}, 1 \mathrm{H})$, $4.78(\mathrm{t}, \mathrm{J}=5.4,1 \mathrm{H}), 2.00-1.94(\mathrm{~m}, 2 \mathrm{H}), 1.73(\mathrm{~s}, 3 \mathrm{H}), 1.57-1.48(\mathrm{~m}, 1 \mathrm{H}), 1.44-1.34(\mathrm{~m}, 1 \mathrm{H})$, 0.15 (s, 3H), 0.13, (s, 3H); ${ }^{13} \mathrm{CNMR}\left(\mathrm{CDCl}_{3}, 100 \mathrm{MHz}\right) \delta$ 143.4, 141.3, 140.1, 137.2, 114.76, 113.25, 80.0, 35.4, 28.0, 19.2, 0.0, -0.8; FTIR (Thin film) $v \max 3078,2998,2970,2923,2850$, 1641, 1616, 1439, 1372, 1313, 1286, 1251, 1200, 1098, $1056 \mathrm{~cm}^{-1}$; HRMS (El $70 \mathrm{eV}$ ) calcd for $\mathrm{C}_{12} \mathrm{H}_{20} \mathrm{OSi} \mathrm{M}^{+} 208.1283 \mathrm{~m} / \mathrm{z}$ found $208.1292 \mathrm{~m} / \mathrm{z}$.

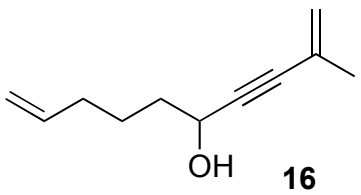

2-Methyldeca-1,9-dien-3-yn-5-ol (16). To a solution of oxalyl chloride $(0.95 \mathrm{~mL}, 11$ mmol) in $\mathrm{CH}_{2} \mathrm{Cl}_{2}(25 \mathrm{~mL})$ at $-78{ }^{\circ} \mathrm{C}$ was added dimethylsulfoxide $(1.7 \mathrm{~mL}, 24 \mathrm{mmol})$ in $\mathrm{CH}_{2} \mathrm{Cl}_{2}$ (5 mL), followed by 6-hexen-1-ol (14) $(1.2 \mathrm{~mL}, 10 \mathrm{mmol})$ in $\mathrm{CH}_{2} \mathrm{Cl}_{2}(10 \mathrm{~mL})$. The mixture was stirred for $5 \mathrm{~min}$, then $\mathrm{NEt}_{3}(7 \mathrm{~mL}, 50 \mathrm{mmol})$ was added dropwise. The solution was stirred at $-78{ }^{\circ} \mathrm{C}$ for $10 \mathrm{~min}$ then warmed to room temperature. The mixture was then diluted with $\mathrm{CH}_{2} \mathrm{Cl}_{2}$ and quenched with water. The aqueous layer was extracted with $\mathrm{CH}_{2} \mathrm{Cl}_{2}$, and the combined organic layers were washed twice with $1 \mathrm{M} \mathrm{HCl}$ (aq.) then brine. The organic layer was then dried over anhydrous $\mathrm{Na}_{2} \mathrm{SO}_{4}$ and concentrated in vacuo. The resulting oil was used without further purification.

To a solution of 2-methylbut-1-en-3-yne $(1 \mathrm{~mL}, 11.5 \mathrm{mmol})$ in THF $(10 \mathrm{~mL})$ at $0{ }^{\circ} \mathrm{C}$ was added $n$-BuLi $(4.45 \mathrm{~mL}, 11 \mathrm{mmol}, 2.5 \mathrm{M}$ in hexanes). This solution was stirred for $15 \mathrm{~min}$ followed by addition of the crude aldehyde from the preceding step. The solution was allowed to warm to room temperature slowly over $1.5 \mathrm{~h}$ at which time it was diluted with diethyl $\mathrm{Et}_{2} \mathrm{O}$ and quenched with saturated aqueous ammonium chloride. The aqueous layer was extracted with $\mathrm{Et}_{2} \mathrm{O}$, the combined organic layers were washed with brine, dried over 
anhydrous $\mathrm{Na}_{2} \mathrm{SO}_{4}$ and concentrated in vacuo. The resulting orange oil was purified by column chromatography $\left(9: 1\right.$ hexanes/ $\left./ \mathrm{Et}_{2} \mathrm{O}\right)$ yielding $1.08 \mathrm{~g}(66 \%)$ of 12 as a pale yellow oil: ${ }^{1} \mathrm{HNMR}\left(\mathrm{CDCl}_{3}, 400 \mathrm{MHz}\right) \delta 5.74(\mathrm{ddt}, \mathrm{J}=16.8,10.0,6.8 \mathrm{~Hz}, 1 \mathrm{H}), 5.21(\mathrm{~s}, 1 \mathrm{H}), 5.15(\mathrm{~s}, 1 \mathrm{H})$, $4.96(\mathrm{~d}, \mathrm{~J}=16.8 \mathrm{~Hz}, 1 \mathrm{H}), 4.90(\mathrm{~d}, \mathrm{~J}=10.0 \mathrm{~Hz}, 1 \mathrm{H}), 4.42(\mathrm{q}, \mathrm{J}=5.9 \mathrm{~Hz}, 1 \mathrm{H}), 3.17$ (d, J = 4.8 $\mathrm{Hz}, 1 \mathrm{H}), 2.04(\mathrm{q}, \mathrm{J}=4.7 \mathrm{~Hz}, 2 \mathrm{H}), 1.81(\mathrm{~s}, 1 \mathrm{H}), 1.74-1.68(\mathrm{~m}, 2 \mathrm{H}), 1.66-1.44(\mathrm{~m}, 2 \mathrm{H}) ;{ }^{13} \mathrm{CNMR}$ $\left(\mathrm{CDCl}_{3}, 400 \mathrm{MHz}\right) \delta 138.4,126.3,121.8,114.7,92.9,89.3,62.4,37.1,33.3,24.4,23.1$; FTIR (Thin film) $v \max 3339$ (br), 3097, 3077, 2977, 2923, 2862, 2223, 1804, 1641, 1615, 1438, 1416, 1374, 1336, 1287, 1124, 1066, 1009, 989, 899, 830, 749, $729 \mathrm{~cm}^{-1}$; HRMS (El $70 \mathrm{eV}$ ) calcd for $\mathrm{C}_{11} \mathrm{H}_{16} \mathrm{O}[\mathrm{M}-\mathrm{H}]^{+} 162.1123 \mathrm{~m} / \mathrm{z}$ found $163.1130 \mathrm{~m} / \mathrm{z}$.

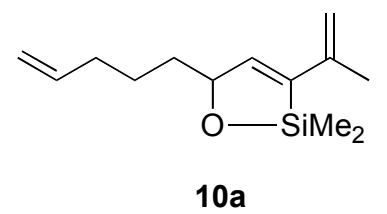

2,2-Dimethyl-5-(pent-4-enyl)-3-(prop-1-en-2-yl)-2,5-dihydro-1,2-oxasilole (10a). To $16(4.94 \mathrm{~g}, 30 \mathrm{mmol})$ was added tetramethyldisilazane $(3.1 \mathrm{~mL}, 18 \mathrm{mmol})$. The solution was stirred for $12 \mathrm{~h}$, then the volatile material was removed in vacuo. The resulting yellow oil was dissolved in THF (100 mL), and a solution of KOt-Bu $(330 \mathrm{mg}, 3 \mathrm{mmol})$ in THF $(15 \mathrm{~mL})$ was added dropwise. The solution was stirred for 1 hour, then diluted with $\mathrm{Et}_{2} \mathrm{O}$ and quenched with saturated aqueous ammonium chloride. The aqueous layer was extracted with $\mathrm{Et}_{2} \mathrm{O}$. The combined organic layers were then washed with brine, dried over anhydrous $\mathrm{Na}_{2} \mathrm{SO}_{4}$ and concentrated in vacuo. The crude product was purified by column chromatography (1\% $\mathrm{NEt}_{3}$ in hexanes) to yield $6.25 \mathrm{~g}$ (93\%) of $10 \mathrm{a}$ as a pale yellow oil: ${ }^{1} \mathrm{HNMR}\left(\mathrm{CDCl}_{3}, 400 \mathrm{MHz}\right)$ $\delta 6.51(\mathrm{~d}, \mathrm{~J}=1.8 \mathrm{~Hz}, 1 \mathrm{H}), 5.81$ (ddt, J = 17.1, 12, $6.6 \mathrm{~Hz}, 1 \mathrm{H}), 5.01$ (dq, J = 17.1, 1.7 Hz, 1H), $5.00(\mathrm{~s}, 1 \mathrm{H}), 4.95$ (ddt, J = 12, 2.5, $1.2 \mathrm{~Hz}, 1 \mathrm{H}), 4.82(\mathrm{~s}, 1 \mathrm{H}), 4.77$ (t, J = $4.7 \mathrm{~Hz}, 1 \mathrm{H}), 2.11-$ $2.06(\mathrm{~m}, 2 \mathrm{H}), 1.92(\mathrm{~s}, 3 \mathrm{H}), 1.67-1.59(\mathrm{~m}, 1 \mathrm{H}), 1.55-1.46(\mathrm{~m}, 3 \mathrm{H}) ;{ }^{13} \mathrm{CNMR}\left(\mathrm{CDCl}_{3}, 400 \mathrm{M} \mathrm{Hz}\right)$ $\delta$ 143.6, 141.1, 140.1, 137.4, 114.7, 113.2, 80.4, 35.6, 32.4, 30.6, 23.1, 19.2, 0.0, -0.8; FTIR (Thin film) $v \max 3078,2999,2970,2940,2859,1982,1935,1824,1773,1641,1617,1571$, 1456, 1539, 1416, 1372, 1313, 1284, 1251, 1198, 1100, 1057, 1035, 994, 971, 933, 910, 881, $820,741,689,676,636 \mathrm{~cm}^{-1}$; HRMS (El $70 \mathrm{eV}$ ) calcd for $\mathrm{C}_{13} \mathrm{H}_{22} \mathrm{OSi} \mathrm{M}^{+} 222.1140 \mathrm{~m} / \mathrm{z}$ found $222.1450 \mathrm{~m} / \mathrm{z}$. 
Cross Metathesis, General Procedure A:

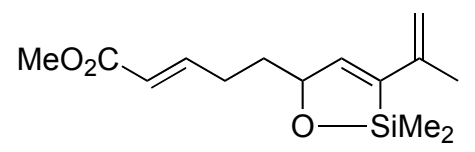

$7 b$

(E)-Methyl-5-(2,2-dimethyl-3-(prop-1-en-2-yl)-2,5-dihydro-1,2-oxasilol-5-yl)-pent-2enoate (7b). To a solution of $7 \mathrm{a}(200 \mathrm{mg}, 0.96 \mathrm{mmol})$ in $\mathrm{CH}_{2} \mathrm{Cl}_{2}(4 \mathrm{~mL})$ was added methyl acrylate $(0.17 \mathrm{~mL}, 1.9 \mathrm{mmol})$, then Hoveyda catalyst $17(30 \mathrm{mg}, 0.048 \mathrm{mmol})$ dissolved in $\mathrm{CH}_{2} \mathrm{Cl}_{2}(1 \mathrm{~mL})$. The resulting solution thoroughly degassed by 5 vacuum/argon backfills, then heated to $40{ }^{\circ} \mathrm{C}$ for $16 \mathrm{~h}$. The mixture was concentrated in vacuo and purified by column chromatography ( $1 \% \mathrm{NEt}_{3}, 3 \% \mathrm{Et}_{2} \mathrm{O}$ in hexanes) to yield $243 \mathrm{mg}(94 \%)$ of the $\mathbf{7 b}$ as a clear colorless oil: ${ }^{1} \mathrm{HNMR}\left(\mathrm{CDCl}_{3}, 400 \mathrm{MHz}\right) \delta 6.95(\mathrm{dt}, \mathrm{J}=15.6,6.8 \mathrm{~Hz}, 1 \mathrm{H}), 6.43(\mathrm{~d}, \mathrm{~J}=1.6 \mathrm{~Hz}$, $1 \mathrm{H}), 5.80,(\mathrm{dt}, \mathrm{J}=15.6,1.4 \mathrm{~Hz}, 1 \mathrm{H}), 4.96(\mathrm{~s}, 1 \mathrm{H}), 4.78(\mathrm{~s}, 1 \mathrm{H}), 4.73(\mathrm{t}, \mathrm{J}=5.2 \mathrm{~Hz}, 1 \mathrm{H}), 3.66$ (s, 3H), 2.3-2.23 (m, 2H), $1.86(\mathrm{~s}, 3 \mathrm{H}), 1.80-1.70(\mathrm{~m}, 1 \mathrm{H}), 1.58-1.49(\mathrm{~m}, 1 \mathrm{H}), 0.29(\mathrm{~s}, 3 \mathrm{H})$, 0.26 (s, 3H); ${ }^{13} \mathrm{CNMR}\left(\mathrm{CDCl}_{3}, 400 \mathrm{MHz}\right) \delta 167.0,149.1,144.1,143.2,141.2,140.6,121.3$, 113.7, 80.81, 51.3, 35.3, 27.6, 20.4, 1.2, 0.4; FTIR (Thin film) $v$ max 3078, 2997, 2951, 2853, 2730, 2650, 2113, 2070, 1939, 1728, 1658, 1616, 1579, 1516, 1497, 1436, 1373, 1315, 1273 , 1251, 1229, 1203, 1169, 1101, 1041, 981, 910, 867, 822, 788, 745, 723, 685, 623, $575 \mathrm{~cm}^{-1}$; HRMS (ESI) calcd for $\mathrm{C}_{14} \mathrm{H}_{22} \mathrm{O}_{2} \mathrm{Si}[\mathrm{M}+\mathrm{Na}]^{+} 273.1287 \mathrm{~m} / \mathrm{z}$ found $273.1287 \mathrm{~m} / \mathrm{z}$.

\section{Cross Metathesis, General Procedure B:}

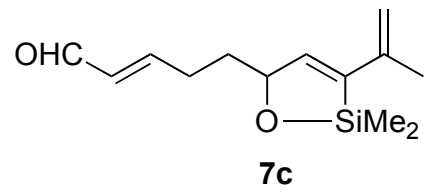

(E)-5-(2,2-Dimethyl-3-(prop-1-en-2-yl)-2,5-dihydro-1,2-oxasilol-5-yl)-pent-2-enal

(7c). To a solution of $7 \mathrm{a}(200 \mathrm{mg}, 0.96 \mathrm{mmol})$ in $\mathrm{CH}_{2} \mathrm{Cl}_{2}(4 \mathrm{~mL})$ was added acrolein $(190 \mu \mathrm{L}$, $2.8 \mathrm{mmol}$ ), 1,4-benzoquinone (10 $\mathrm{mg}, 0.092 \mathrm{mmol})$, then Hoveyda catalyst (17) $(30 \mathrm{mg}$, $0.048 \mathrm{mmol})$ dissolved in $\mathrm{CH}_{2} \mathrm{Cl}_{2}(1 \mathrm{~mL})$. The resulting solution was thoroughly degassed with 5 vacuum/argon backfills, then heated to $40^{\circ} \mathrm{C}$ for $18 \mathrm{~h}$. The mixture was then concentrated in vacuo and purified by column chromatography $\left(1 \% \mathrm{NEt}_{3}, 3 \% \mathrm{Et}_{2} \mathrm{O}\right.$ in hexanes) to yield $219 \mathrm{mg}$ ( $85 \%)$ of $7 \mathrm{c}$ as a pale yellow oil: ${ }^{1} \mathrm{HNMR}\left(\mathrm{CDCl}_{3}, 400 \mathrm{MHz}\right) \delta 9.51$ $(\mathrm{d}, \mathrm{J}=8.8 \mathrm{~Hz}, 1 \mathrm{H}), 6.90(\mathrm{dt}, \mathrm{J}=15.6,6.8 \mathrm{~Hz}, 1 \mathrm{H}), 6.48(\mathrm{~s}, 1 \mathrm{H}), 6.14(\mathrm{dd}, \mathrm{J}=15.6,8.8 \mathrm{~Hz}$, $1 \mathrm{H}), 5.03(\mathrm{~s}, 1 \mathrm{H}), 4.84(\mathrm{~s}, 1 \mathrm{H}), 4.81(\mathrm{t}, \mathrm{J}=5.6 \mathrm{~Hz}, 1 \mathrm{H}), 2.51-2.38(\mathrm{~m}, 2 \mathrm{H}), 1.92(\mathrm{~s}, 3 \mathrm{H}), 1.91-$ 
$1.83(\mathrm{~m}, 1 \mathrm{H}), 1.76-1.60(\mathrm{~m}, 1 \mathrm{H}), 0.35(\mathrm{~s}, 3 \mathrm{H}), 0.33(\mathrm{~s}, 3 \mathrm{H}) ;{ }^{13} \mathrm{CNMR}\left(\mathrm{CDCl}_{3}, 400 \mathrm{MHz}\right) \delta$ 193.5, 158.1, 143.3, 140.7, 132.6, 116.1, 80.3, 34.9, 28.0, 20.0, 0.8, 0.0; FTIR (Thin film) $v$ $\max 3078,2921,2850,2731,1693,1637,1617,1541,1439,1373,1313,1286,1251,1174$, 1133, 1101, 1056, 974, 927, 910, 867, 824, 788, 745, $670 \mathrm{~cm}^{-1}$; HRMS (ESI) calcd for $\mathrm{C}_{13} \mathrm{H}_{20} \mathrm{O}_{2} \mathrm{Si}[\mathrm{M}+\mathrm{Na}]^{+} 259.1130 \mathrm{~m} / \mathrm{z}$ found $259.1133 \mathrm{~m} / \mathrm{z}$.

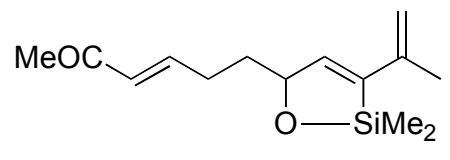

7d

(E)-6-(2,2-Dimethyl-3-(prop-1-en-2-yl)-2,5-dihydro-1,2-oxasilol-5-yl)-hex-3-en-2-

one (7d). General procedure A was followed with 7a (300 $\mathrm{mg}, 1.44 \mathrm{mmol})$, methyl vinyl ketone $(0.35 \mathrm{~mL}, 4.3 \mathrm{mmol})$ and catalyst $17(45 \mathrm{mg}, 0.07 \mathrm{mmol})$. Purification of the crude product by column chromatography ( $1 \% \mathrm{NEt}_{3}, 3 \% \mathrm{Et}_{2} \mathrm{O}$ in hexanes) yielded $331 \mathrm{mg}$ (92\%) of $\mathbf{7 d}$ as a pale yellow oil: ${ }^{1} \mathrm{HNMR}\left(\mathrm{CDCl}_{3}, 400 \mathrm{MHz}\right) \delta 6.84(\mathrm{dt}, \mathrm{J}=16,6.8 \mathrm{~Hz}, 1 \mathrm{H}), 6.48(\mathrm{~s}$, $1 \mathrm{H}), 6.1(\mathrm{~d}, \mathrm{~J}=16 \mathrm{~Hz}, 1 \mathrm{H}), 5.02(\mathrm{~s}, 1 \mathrm{H}), 4.83(\mathrm{~s}, 1 \mathrm{H}), 4.80(\mathrm{t}, \mathrm{J}=4.6), 2.4-2.29(\mathrm{~m}, 2 \mathrm{H}), 2.14$ $(\mathrm{s}, 3 \mathrm{H}), 1.92(\mathrm{~s}, 3 \mathrm{H}), 1.87-1.79(\mathrm{~m}, 1 \mathrm{H}), 1.65-1.56(\mathrm{~m}, 1 \mathrm{H}), 0.33(\mathrm{~s}, 3 \mathrm{H}), 0.31(\mathrm{~s}, 3 \mathrm{H})$; ${ }^{13} \mathrm{CNMR}\left(\mathrm{CDCl}_{3}, 400 \mathrm{MHz}\right) \delta 197.3,146.8,142.7,142.1,140.0,130.1,115.2,79.6,34.4$, 26.9, 25.6, 19.2, 0.0, -0.8; FTIR (Thin film) $v \max 3078,3000,2951,2923,2853,2729,2651$, 2488, 2168, 2043, 1935, 1771, 1698, 1627, 1571, 1455, 1437, 1361, 1314, 1286, 1252, 1191, 1169, 1101, 1056, 980, 964, 910, 867, 825, 788, 745, 689, 676, 605, 577, $545 \mathrm{~cm}^{-1}$; HRMS (ESI) calcd for $\mathrm{C}_{14} \mathrm{H}_{22} \mathrm{O}_{3} \mathrm{Si}[\mathrm{M}+\mathrm{H}]^{+} 267.1416 \mathrm{~m} / \mathrm{z}$ found $267.1416 \mathrm{~m} / \mathrm{z}$.

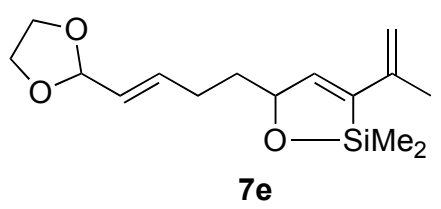

(E)-5-(4-(1,3-Dioxolan-2-yl)but-3-enyl)-2,2-dimethyl-3-(prop-1-en-2-yl)-2,5-dihydro1,2-oxasilole (7e). General procedure B was followed with $7 \mathrm{a}(200 \mathrm{mg}, 0.96 \mathrm{mmol})$, with acrolein ethylene acetal $(0.29 \mathrm{~mL}, 3.2 \mathrm{mmol}), 1,4$-benzoquinone $(10 \mathrm{mg}, 0.092 \mathrm{mmol})$ and catalyst $17(30 \mathrm{mg}, 0.048 \mathrm{mmol})$. Purification of the crude product by column chromatography ( $1 \% \mathrm{NEt}_{3}, 2 \% \mathrm{Et}_{2} \mathrm{O}$ in hexanes) gave $219 \mathrm{mg}(81 \%)$ of $7 \mathrm{e}$ as a clear yellow oil: ${ }^{1} \mathrm{HNMR}\left(\mathrm{CDCl}_{3}, 400 \mathrm{MHz}\right) \delta 6.48(\mathrm{~d}, \mathrm{~J}=1.6 \mathrm{~Hz}, 1 \mathrm{H}), 5.97(\mathrm{dt}, \mathrm{J}=15.2,6.8 \mathrm{~Hz}$, 1H), 5.49 (dd, J = 15.2, $6.4 \mathrm{~Hz}, 1 \mathrm{H}), 5.19(\mathrm{~d}, \mathrm{~J}=6.4 \mathrm{~Hz}, 1 \mathrm{H}), 5.00(\mathrm{~s}, 1 \mathrm{H}), 4.82(\mathrm{~s}, 1 \mathrm{H}), 4.77$ $(\mathrm{t}, \mathrm{J}=5.4 \mathrm{~Hz}, 1 \mathrm{H}), 4.00-3.86(\mathrm{~m}, 4 \mathrm{H}), 2.25-2.14(\mathrm{~m}, 2 \mathrm{H}), 1.9(\mathrm{~s}, 3 \mathrm{H}), 1.80-1.70(\mathrm{~m}, 1 \mathrm{H}), 1.61-$ 
$1.52(\mathrm{~m}, 1 \mathrm{H}), 0.33(\mathrm{~s}, 3 \mathrm{H}), 0.31(\mathrm{~s}, 3 \mathrm{H}) ;{ }^{13} \mathrm{CNMR}\left(\mathrm{CDCl}_{3}, 400 \mathrm{MHz}\right) \delta 144.5,142.8,141.4$, 137.3, 126.5, 116.1, 104.1, 81.1, 64.9, 36.3, 27.7, 20.5, 1.3, 0.5; FTIR (Thin film) $v$ max3078, 2950, 2922, 2884, 2765, 2678, 1938, 1775, 1743, 1676, 1616, 1571, 1474, 1440, 1399, 1373, 1346, 1313, 1286, 1251, 1211, 1152, 1116, 1103, 1056, 960, 911, 867, 824, 787, 742, 690, $676,599 \mathrm{~cm}^{-1}$; HRMS (ESI) calcd for $\mathrm{C}_{15} \mathrm{H}_{24} \mathrm{O}_{3} \mathrm{Si}[\mathrm{M}+\mathrm{Na}]^{+} 303.1392 \mathrm{~m} / \mathrm{z}$ found $303.1394 \mathrm{~m} / \mathrm{z}$.

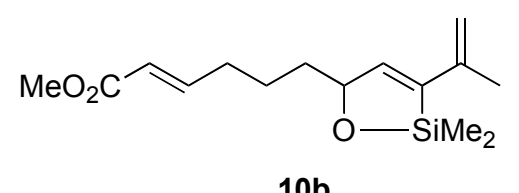

(E)-Methyl-6-(2,2-dimethyl-3-(prop-1-en-2-yl)-2,5-dihydro-1,2-oxasilol-5-yl)-hex-2enoate (10b). General procedure A was followed with $10 \mathrm{a}(200 \mathrm{mg}, 0.9 \mathrm{mmol})$, methyl acrylate $(0.245 \mathrm{~mL}, 2.7 \mathrm{mmol})$ and catalyst $17(30 \mathrm{mg}, 0.048 \mathrm{mmol})$. Purification of the crude product by column chromatography (1\% NEt 3 , $3 \% \mathrm{Et}_{2} \mathrm{O}$ in hexanes) gave $240 \mathrm{mg}$ (94 \%) of $10 \mathrm{~b}$ as a clear colorless oil: ${ }^{1} \mathrm{HNMR}\left(\mathrm{CDCl}_{3}, 400 \mathrm{MHz}\right) \delta 6.96(\mathrm{dt}, \mathrm{J}=15.6,6.9 \mathrm{~Hz}, 1 \mathrm{H})$, $6.48(\mathrm{~d}, \mathrm{~J}=1.8 \mathrm{~Hz}, 1 \mathrm{H}), 5.83(\mathrm{dt}, \mathrm{J}=15.6,1.6 \mathrm{~Hz}, 1 \mathrm{H}), 5.00(\mathrm{~s}, 1 \mathrm{H}), 4.82(\mathrm{~s}, 1 \mathrm{H}), 4.76(\mathrm{t}, \mathrm{J}=$ $4.6 \mathrm{~Hz}, 1 \mathrm{H}), 3.72(\mathrm{~s}, 3 \mathrm{H}), 2.24(\mathrm{q}, \mathrm{J}=7.2,2 \mathrm{H}), 1.93(\mathrm{~s}, 3 \mathrm{H}), 1.68-1.43(\mathrm{~m}, 4 \mathrm{H}), 0.33(\mathrm{~s}, 3 \mathrm{H})$, 0.31 (s, 3H); ${ }^{13} \mathrm{CNMR}\left(\mathrm{CDCl}_{3}, 100 \mathrm{MHz}\right) \delta 166.6,148.8,144.0,142.3,140.9,120.6,15.7$, 81.0, 50.9, 36.3, 36.3, 31.7, 23.1, 20.0, 0.8, 0.0; ; FTIR (Thin film) $v$ max 3078, 2997, 2949, 2856, 2730, 2649, 2108, 1934, 1728, 1659, 1616, 1572, 1456, 1436, 1373, 1315, 1271, 1251 , 1199, 1175, 1157, 1104, 1036, 9779, 911, 879, 822, 788, 756, 741, 719, 690, 676, $623 \mathrm{~cm}^{-1}$; HRMS (ESI) calcd for $\mathrm{C}_{13} \mathrm{H}_{20} \mathrm{O}_{2} \mathrm{Si}[\mathrm{M}+\mathrm{H}]^{+} 281.1573 \mathrm{~m} / \mathrm{z}$ found $281.1562 \mathrm{~m} / \mathrm{z}$.

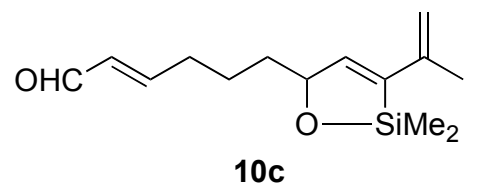

(E)-6-(2,2-Dimethyl-3-(prop-1-en-2-yl)-2,5-dihydro-1,2-oxasilol-5-yl)-hex-2-enal (10c). General procedure B was followed with 10a $(200 \mathrm{mg}, 0.9 \mathrm{mmol}$, acrolein ( $270 \mu \mathrm{L}, 4.0$ $\mathrm{mmol}$ ), 1,4-benzoquinone (10 mg, $0.092 \mathrm{mmol}$ ) and catalyst $17(30 \mathrm{mg}, 0.048 \mathrm{mmol})$. Purifcation of the crude product by column chromatography ( $1 \% \mathrm{NEt}_{3}, 3 \% \mathrm{Et}_{2} \mathrm{O}$ in hexanes) yielded $182 \mathrm{mg}(81 \%)$ of $10 \mathrm{c}$ as a pale yellow oil: ${ }^{1} \mathrm{HNMR}\left(\mathrm{CDCl}_{3}, 400 \mathrm{MHz}\right) \delta 9.48(\mathrm{~d}, \mathrm{~J}=$ $7.6 \mathrm{~Hz}, 1 \mathrm{H}$ ), $6.83(\mathrm{dt}, \mathrm{J}=15.6,6.8 \mathrm{~Hz}, 1 \mathrm{H}), 6.46(\mathrm{~d}, \mathrm{~J}=1.6 \mathrm{~Hz}, 1 \mathrm{H}), 6.10$ (ddt, J = 15.6, 7.6, $1.2 \mathrm{~Hz}, 1 \mathrm{H}), 4.991(\mathrm{~s}, 1 \mathrm{H}), 4.81(\mathrm{~s}, 1 \mathrm{H}), 4.754(\mathrm{t}, \mathrm{J}=3.8 \mathrm{~Hz}, 1 \mathrm{H}), 2.37$ (q, J = $6.9 \mathrm{~Hz}, 2 \mathrm{H}$ ), 
$1.90(\mathrm{~s}, 3 \mathrm{H}), 1.72-1.37(\mathrm{~m}, 4 \mathrm{H}), 0.33(\mathrm{~s}, 3 \mathrm{H}), 0.31(\mathrm{~s}, 3 \mathrm{H}) ;{ }^{13} \mathrm{CNMR}\left(\mathrm{CDCl}_{3}, 100 \mathrm{MHz}\right) \delta$ 194.0, 158.3, 144.3, 142.9, 141.3, 133.1, 116.3, 81.3, 36.7, 32.7, 29.7, 23.3, 20.4, 1.3, 0.4; FTIR (Thin film) $v \max 3078,2996,2944,2852,2732,1694,1677,1616,1456,1438,1373,1314$, $1251,1168,1133,1100,1056,1034,975,929,910,875,822,788,741,676 \mathrm{~cm}^{-1}$; HRMS (ESI) calcd for $\mathrm{C}_{14} \mathrm{H}_{22} \mathrm{O}_{2} \mathrm{Si}[\mathrm{M}+\mathrm{Na}]^{+} 273.1287 \mathrm{~m} / \mathrm{z}$ found $273.1292 \mathrm{~m} / \mathrm{z}$.

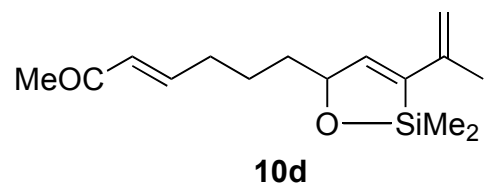

(E)-7-(2,2-Dimethyl-3-(prop-1-en-2-yl)-2,5-dihydro-1,2-oxasilol-5-yl)hept-3-en-2-

one. (10d) General procedure A was followed with 10a (150 $\mathrm{mg}, 0.67 \mathrm{mmol})$, methyl vinyl ketone $(0.170 \mathrm{~mL}, 2 \mathrm{mmol})$ and catalyst $17(23 \mathrm{mg}, 0.037 \mathrm{mmol})$. Purification of the crude product by column chromatography ( $1 \% \mathrm{NEt}_{3}, 3 \% \mathrm{Et}_{2} \mathrm{O}$ in hexanes) yielded $158 \mathrm{mg}(88 \%)$ of the $10 \mathrm{~d}$ as a clear colorless oil: ${ }^{1} \mathrm{HNMR}\left(\mathrm{CDCl}_{3}, 400 \mathrm{MHz}\right) \delta 6.79(\mathrm{dt}, \mathrm{J}=15.6,7.2 \mathrm{~Hz}, 1 \mathrm{H})$, $6.48(\mathrm{~d}, \mathrm{~J}=1.6 \mathrm{~Hz}, 1 \mathrm{H}), 6.08(\mathrm{~d}, \mathrm{~J}=15.6 \mathrm{~Hz}, 1 \mathrm{H}), 5.01(\mathrm{~s}, 1 \mathrm{H}), 4.83(\mathrm{~s}, 1 \mathrm{H}), 4.77(\mathrm{t}, \mathrm{J}=4.6$ $\mathrm{Hz}, 1 \mathrm{H}), 2.26(\mathrm{q}, \mathrm{J}=7.2 \mathrm{~Hz}, 1 \mathrm{H}), 2.24(\mathrm{~s}, 3 \mathrm{H}), 1.92(\mathrm{~s}, 3 \mathrm{H}), 1.71-1.49(\mathrm{~m}, 4 \mathrm{H}), 0.33(\mathrm{~s}, 3 \mathrm{H})$, 0.31 (s, 3H); ${ }^{13} \mathrm{CNMR}\left(\mathrm{CDCl}_{3}, 100 \mathrm{MHz}\right) \delta$ 198.6, 148.0, 144.5, 142.8, 141.3, 131.5, 116.2, 81.4, 36.9, 32.4, 26.8, 23.7, 20.5, 1.3, 0.5 ; FTIR (Thin film) $v$ max 3078, 3000, 2943, 2851, 1698, 1676, 1627, 1456, 1436, 1361, 1315, 1252, 1185, 1162, 1103, 1057, 1033, 979, 910 , $879,822,788,675 \mathrm{~cm}^{-1}$; HRMS (ESI) calcd for $\mathrm{C}_{15} \mathrm{H}_{24} \mathrm{O}_{2} \mathrm{Si}[\mathrm{M}+\mathrm{Na}]^{+} 287.1443 \mathrm{~m} / \mathrm{z}$ found $259.1431 \mathrm{~m} / \mathrm{z}$.

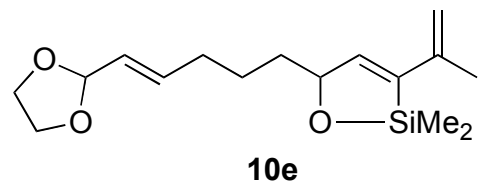

(E)-5-(5-(1,3-Dioxolan-2-yl)pent-4-enyl)-2,2-dimethyl-3-(prop-1-en-2-yl)-

2,5-dihydro-1,2-oxasilole (10e). General procedure B was followed with 10a (200 mg, 0.9 $\mathrm{mmol})$, acrolein ethylene acetal $(0.27 \mathrm{~mL}, 2.7 \mathrm{mmol}), 1,3$-benzoquinone (10 $\mathrm{mg}, 0.092 \mathrm{mmol})$ and catalyst 17 (30 mg, $0.048 \mathrm{mmol})$. Purification of the crude product by column chromatography (1\% $\mathrm{NEt}_{3}, 2 \% \mathrm{Et}_{2} \mathrm{O}$ in hexanes) yielded $188 \mathrm{mg}(71 \%)$ of $\mathbf{3 e}$ as a clear colorless oil: ${ }^{1} \mathrm{HNMR}\left(\mathrm{CDCl}_{3}, 400 \mathrm{MHz}\right) \delta 6.49(\mathrm{~s}, 1 \mathrm{H}), 5.89(\mathrm{dt}, \mathrm{J}=15.6,6.4 \mathrm{~Hz}, 1 \mathrm{H}), 4.56$ (dd, J = 15.6, $6.8 \mathrm{~Hz}, 1 \mathrm{H}$ ), $5.15(\mathrm{~d}, \mathrm{~J}=6.8,1 \mathrm{H}), 4.96(\mathrm{~s}, 1 \mathrm{H}), 4.79(\mathrm{~s}, 1 \mathrm{H}), 3.71(\mathrm{t}, \mathrm{J}=5.2 \mathrm{~Hz}$, 1H), 4.00-3.80 (m, 4H), $2.11(\mathrm{q}, \mathrm{J}=6.6 \mathrm{~Hz}), 1.88(\mathrm{~s}, 3 \mathrm{H}), 1.65-1.41(\mathrm{~m}, 4 \mathrm{H}), 0.30(\mathrm{~s}, 3 \mathrm{H})$, 0.28 (s, 3H); ${ }^{13} \mathrm{CNMR}\left(\mathrm{CDCl}_{3}, 100 \mathrm{MHz}\right) \delta$ 144.7, 142.5, 141.3, 137.3, 126.4, 115.9, 103.0, 
84.5, 64.8, 36.8, 31.9, 24.0, 20.4, 1.2, 0.4; FTIR (Thin film) $v$ max 3077, 2995, 2943, 2885, $2861,1769,1677,1616,1571,1542,1474,1457,1438,1399,1373,1346,1313,1250,1210$, 1150, 1121, 1105, 1060, 1034, 964, 911, 875, 822, 788, 741, 690, $676 \mathrm{~cm}^{-1}$; HRMS (ESI) calcd for $\mathrm{C}_{16} \mathrm{H}_{26} \mathrm{O}_{3} \mathrm{Si}[\mathrm{M}+\mathrm{Na}]^{+} 317.1549 \mathrm{~m} / \mathrm{z}$ found $317.1561 \mathrm{~m} / \mathrm{z}$.

\section{Thermal Intramolecular Diels-Alder Reactions, General Procedure C:}

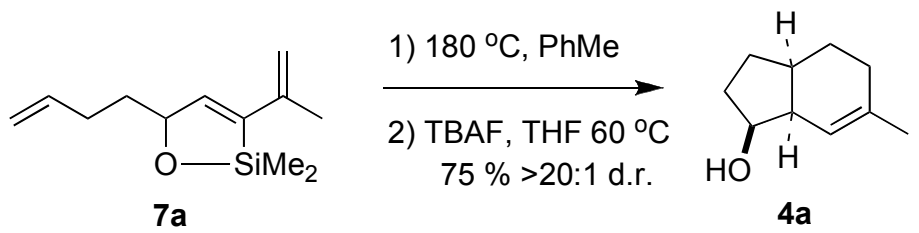

rac-(1S,3aR,7aS)-6-Methyl-2,3,3a,4,5,7a-hexahydro-1H-inden-1-ol (4a). A single crystal of BHT was added to a solution of $7 \mathrm{a}(200 \mathrm{mg}, 0.96 \mathrm{mmol})$ in toluene $(20 \mathrm{~mL})$. The solution was heated to $180{ }^{\circ} \mathrm{C}$ for $\mathrm{h}$ in a resealable tube, then concentrated. The resulting volatile oil was dissolved in THF $(20 \mathrm{~mL})$ and was treated with tetrabutylammonium fluoride trihydrate $(910 \mathrm{mg}, 2.88 \mathrm{mmol})$ at $60{ }^{\circ} \mathrm{C}$ for $1.5 \mathrm{~h}$. The solution was diluted with $\mathrm{Et}_{2} \mathrm{O}$ and quenched with saturated aqueous ammonium chloride. The aqueous layer was extracted with $\mathrm{Et}_{2} \mathrm{O}$, the combined organic layers washed with brine, dried over anhydrous $\mathrm{Na}_{2} \mathrm{SO}_{4}$ and concentrated in vacuo. The crude alcohol was purfied by column chromatography $(6: 1$ pentane to $\left.\mathrm{Et}_{2} \mathrm{O}\right)$ to yield $110 \mathrm{mg}(75 \%)$ of $4 \mathrm{a}$ as a clear yellow oil.: ${ }^{1} \mathrm{HNMR}\left(\mathrm{CDCl}_{3}, 400 \mathrm{MHz}\right)$ $\delta 5.29$ (s, 1H), 4.14 (td, J = 4.8, $2.4 \mathrm{~Hz}, 1 \mathrm{H}$ ), 2.39 (s, 1H), 2.10-2.00 (ddddd, J = 10.8, 8.8, 8.8, 5.2, $5.2 \mathrm{~Hz}, 1 \mathrm{H}), 1.95-1.84(\mathrm{~m}, 4 \mathrm{H}), 1.80-1.68(\mathrm{~m}, 6 \mathrm{H}), 1.59-1.4(\mathrm{~m}, 2 \mathrm{H}) ;{ }^{13} \mathrm{CNMR}\left(\mathrm{CDCl}_{3}\right.$, $100 \mathrm{MHz}$ ) $\delta 141.4,117.9,75.0,45.8,34.5,33.4,29.5,29.4,29.0,24.3$; FTIR (Thin film) $v$ $\max 3393$ (br), 2957, 2918, 1666, 1646, 1484, 1449, 1389, 1364, 1304, 1261, 1245, 1213, $1181,1161,1125,139,1089,1061,1024,996,929,907,864,839,817,804,760,741,669$ $\mathrm{cm}^{-1}$; HRMS (El $70 \mathrm{eV}$ ) calcd for $\mathrm{M}^{+} 152.1201 \mathrm{~m} / \mathrm{z}$ found $152.1201 \mathrm{~m} / \mathrm{z}$

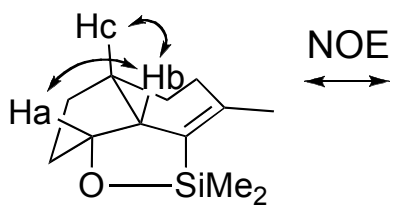




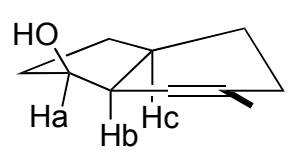

$4 a$

\begin{tabular}{ccc}
\hline Proton & Chemical Shift $(\mathrm{ppm})$ & Multiplicity, $J(\mathrm{~Hz})$ \\
\hline $\mathrm{Ha}$ & 4.14 & $\operatorname{td~} 4.8,2.4$ \\
$\mathrm{Hb}$ & 2.39 & br s \\
$\mathrm{Hc}$ & 2.05 & ddddd $10.8,8.8,8.8,5.2,5.2$
\end{tabular}
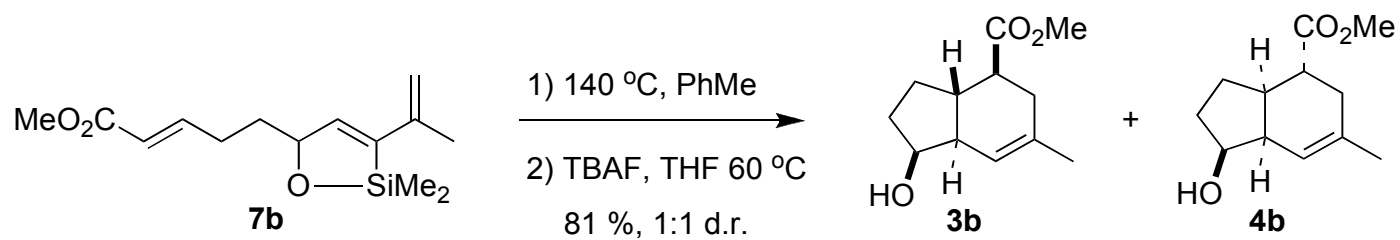

Methyl-1-hydroxy-6-methyl-2,3,3a,4,5,7a-hexahydro-1H-indene-4-carboxylate (3b and $4 \mathrm{~b})$. Following general procedure $C$, a solution of $7 \mathrm{~b}(50 \mathrm{mg}, 0.19 \mathrm{mmol})$ and 1 crystal of $\mathrm{BHT}$ in toluene $(6 \mathrm{~mL})$ was heated to $140{ }^{\circ} \mathrm{C}$ for $20 \mathrm{~h}$, then concentrated. The resulting oil was dissolved in THF $(6 \mathrm{~mL})$ and treated with tetrabutylammonium fluoride trihydrate $(180 \mathrm{mg}$, $0.57 \mathrm{mmol}$ ) at $60{ }^{\circ} \mathrm{C}$ for $1.5 \mathrm{~h}$. Purification of the crude cycloadduct by column chromatography ( $4: 1$ hexanes to ethyl acetate) yielded $8.8 \mathrm{mg} \mathrm{(22 \% )}$ of $3 \mathbf{b}$ as a clear yellow oil, $5.8 \mathrm{mg} \mathrm{(15 \% )}$ of $\mathbf{4 b}$ as a clear yellow oil, and $17.7 \mathrm{mg} \mathrm{(45 \% )}$ of a 1.25:1 mixture of $\mathbf{3 b}$ and $\mathbf{4 b}$. (82\% total).

Data for 3b: ${ }^{1} \mathrm{HNMR}\left(\mathrm{CDCl}_{3}, 400 \mathrm{MHz}\right) \delta 5.57(\mathrm{~s}, 1 \mathrm{H}, 1 \mathrm{H}), 4.27(\mathrm{t}, J=6 \mathrm{~Hz}, 1 \mathrm{H}), 3.70$ (s, 3H), 2.52 (td, $J=10.8,6.8 \mathrm{~Hz}, 1 \mathrm{H}$ ), 2.35 (dd, $J=17.2,10.8 \mathrm{~Hz}, 1 \mathrm{H}$ ), 2.27 (dd, $J=17.2$, $6.8 \mathrm{~Hz}, 1 \mathrm{H}$ ), 2.17 (dddd $J=14.6,10.8,6.0,2.4 \mathrm{~Hz}, 1 \mathrm{H}$ ), 2.06 (qd, $J=10.8,6.4 \mathrm{~Hz}, 1 \mathrm{H}$ ), 1.93$1.84(\mathrm{~m}, 2 \mathrm{H}), 1.71(\mathrm{~s}, 3 \mathrm{H}), 1.65(\mathrm{dt}, J=14.4,8.4 \mathrm{~Hz}, 1 \mathrm{H}), 1.23(\mathrm{qd}, J=11.6,7.6 \mathrm{~Hz}, 1 \mathrm{H})$; ${ }^{13} \mathrm{CNMR}\left(\mathrm{CDCl}_{3}, 100 \mathrm{MHz}\right) \delta 175.8,137.1,119.7,72.4,51.6,50.9,45.4,41.0,34.7,34.4$, 27.0, 23.2; FTIR (Thin film) $v \max 3428,2916,1733,1642,1435,1374,1327,1292,1262$, 1242, 1190, 1169, 1152, 1087, 1020, 913, 847, $743 \mathrm{~cm}^{-1}$; HRMS (ESI) calcd for $\mathrm{C}_{12} \mathrm{H}_{18} \mathrm{O}_{3}$ $[\mathrm{M}+\mathrm{H}]^{+} 211.1334 \mathrm{~m} / \mathrm{z}$ found $211.1328 \mathrm{~m} / \mathrm{z}$.

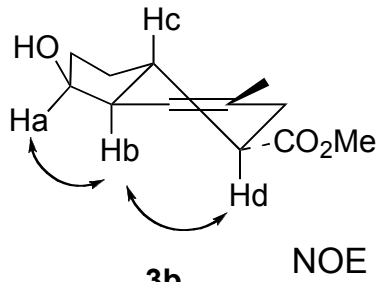

\begin{tabular}{ccc}
\hline Proton & Chemical Shift $(\mathrm{ppm})$ & Multiplicity, $J(\mathrm{~Hz})$ \\
\hline $\mathrm{Ha}$ & 4.27 & $\sim \mathrm{t}, 6$ \\
$\mathrm{Hc}$ & 2.06 & $\mathrm{qd}, 10.8,6.8$ \\
$\mathrm{Hd}$ & 2.52 & $\mathrm{td}, 10.8,6.8$
\end{tabular}


Data for 4b: ${ }^{1} \mathrm{HNMR}\left(\mathrm{CDCl}_{3}, 400 \mathrm{MHz}\right) \delta 5.43(\mathrm{~s}, 1 \mathrm{H}), 4.18(\mathrm{td}, J=4,1.2 \mathrm{~Hz}, 1 \mathrm{H}), 3.69$ (s, 3H), 2.61 (dt, $J=11.2,8 \mathrm{~Hz}, 1 \mathrm{H}), 2.48(\mathrm{br} \mathrm{s}, 1 \mathrm{H}), 2.32(\mathrm{qd}, J=8.8,4.8 \mathrm{~Hz}, 1 \mathrm{H}), 2.13(\mathrm{~d}, J$ $=8 \mathrm{~Hz}, 2 \mathrm{H}), 2.00$ (dddd, 12.8, 8, 8, 3.6Hz, $1 \mathrm{H}), 1.88-1.79(\mathrm{~m}, 1 \mathrm{H}), 1.78(\mathrm{~s}, 3 \mathrm{H}), 1.75-1.59(\mathrm{~m}$, $2 \mathrm{H}), 1.56(\mathrm{~s}, 1 \mathrm{H}) ;{ }^{13} \mathrm{CNMR}\left(\mathrm{CDCl}_{3}, 100 \mathrm{MHz}\right) \delta 176.7,138.8,118.1,74.9,51.6,46.76,45.6$, 36.7, 33.2, 33.1, 28.5, 23.8; FTIR (Thin film) $v \max 3429$ (br), 2961, 1720, 1642, 1432, 1377 , 1311, 1262, 1193, 1165, 1080, 10268, 995, 913, $743 \mathrm{~cm}^{-1}$; HRMS (ESI) calcd for $\mathrm{C}_{12} \mathrm{H}_{18} \mathrm{O}_{3}$ $[\mathrm{M}+\mathrm{H}]^{+} 211.1334 \mathrm{~m} / \mathrm{z}$ found $211.1328 \mathrm{~m} / \mathrm{z}$.

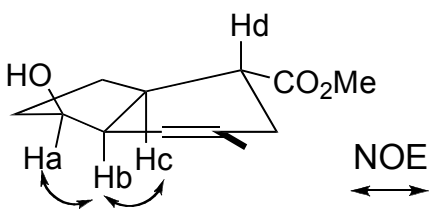

4b

\begin{tabular}{ccc}
\hline Proton & Chemical Shift $(\mathrm{ppm})$ & Multiplicity, $J(\mathrm{~Hz})$ \\
\hline $\mathrm{Ha}$ & 4.17 & $\mathrm{td}, 4,1.2$ \\
$\mathrm{Hb}$ & 2.48 & $\mathrm{br} \mathrm{s}$ \\
$\mathrm{Hc}$ & 2.32 & $\mathrm{qd}, 8.8,4.8$ \\
$\mathrm{Hd}$ & 2.61 & $\mathrm{dt}, 11.2,8$
\end{tabular}

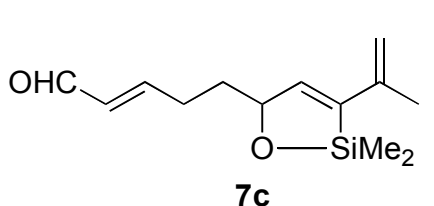

1) $120^{\circ} \mathrm{C}, \mathrm{PhMe}$
2) TBAF, THF $60 \%, 2.5: 1$ d.r.
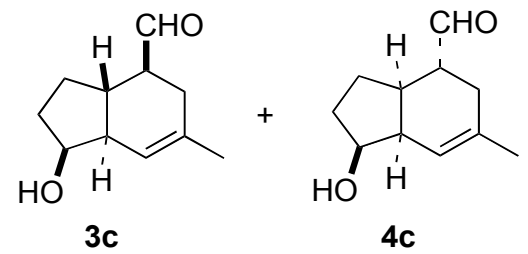

rac-(1S,3aS,4S,7aS)-1-Hydroxy-6-methyl-2,3,3a,4,5,7a-hexahydro-1H-indene-4-

carbaldehyde (3c and $4 c)$. Following general procedure $C$, a solution of triene $7 \mathbf{b}$ (50 $\mathrm{mg}$, $0.19 \mathrm{mmol})$ and 1 crystal of $\mathrm{BHT}$ in toluene $(6 \mathrm{~mL})$ was heated to $120{ }^{\circ} \mathrm{C}$ for $20 \mathrm{~h}$, then concentrated. The resulting oil was dissolved in THF $(2 \mathrm{~mL})$ and tetrabutylammonium fluoride trihydrate $(200 \mathrm{mg}, 0.63 \mathrm{mmol})$ was added. The solution was stirred at ambient temperature for $12 \mathrm{~h}$, then diluted with $\mathrm{Et}_{2} \mathrm{O}$ and quenched with a saturated solution of ammonium chloride. The aqueous layer was extracted with $\mathrm{Et}_{2} \mathrm{O}$, the combined organic layers were washed with brine, dried over anhydrous $\mathrm{Na}_{2} \mathrm{SO}_{4}$, and concentrated in vacuo.. Purification of the crude cycloadduct by column chromatography ( $4: 1$ hexanes to ethyl acetate) yielded $23 \mathrm{mg} \mathrm{(60 \% )}$ of an inseparable $2.5: 1$ mixture of $3 c$ and $\mathbf{4 c}$ clear yellow oil. 


\section{Lewis Acid Promoted Intramolecular Diels-Alder Reactions, General Procedure D:}

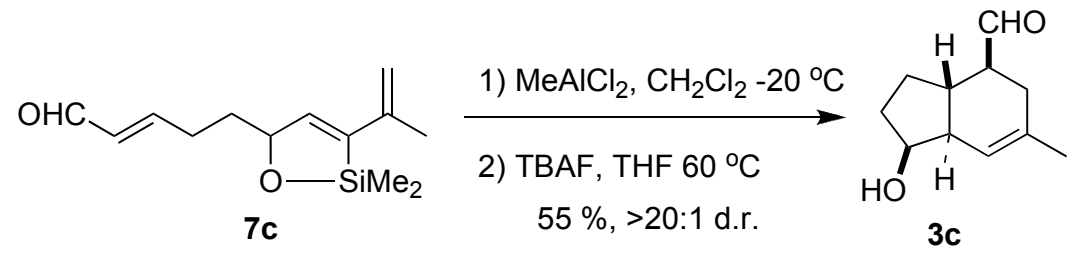

To a solution of $7 \mathrm{c}(50 \mathrm{mg}, 0.21 \mathrm{mmol})$ in $\mathrm{CH}_{2} \mathrm{Cl}_{2}(21 \mathrm{~mL}, 0.01 \mathrm{M})$ at $-78{ }^{\circ} \mathrm{C}$ was added $\mathrm{MeAICl}_{2}\left(210 \mu \mathrm{L}, 0.21 \mathrm{mmol}, 1 \mathrm{M}\right.$ in $\left.\mathrm{CH}_{2} \mathrm{Cl}_{2}\right)$ over $5 \mathrm{~min}$. The solution was then immediately warmed to $-20{ }^{\circ} \mathrm{C}$ and stirred for $3 \mathrm{~h}$. The reaction mixture was quenched with a saturated aqueous solution of Rochelle's salt and warmed to room temperature. The aqueous layer was extracted with $\mathrm{CH}_{2} \mathrm{Cl}_{2}$. The combined organic layers were washed with brine, dried over anhydrous $\mathrm{Na}_{2} \mathrm{SO}_{4}$, and concentrated in vacuo. The crude cycloadduct was then dissolved in THF (2 mL) and tetrabutylammonium fluoride trihydrate $(200 \mathrm{mg}, 0.63 \mathrm{mmol})$ was added. The solution was stirred for $12 \mathrm{~h}$ at room temperature, then diluted with $\mathrm{Et}_{2} \mathrm{O}$ and quenched with a saturated solution of ammonium chloride. The aqueous layer was extracted with $\mathrm{Et}_{2} \mathrm{O}$, the combined organic layers were washed with brine, dried over anhydrous $\mathrm{Na}_{2} \mathrm{SO}_{4}$, and concentrated in vacuo. Purification of the crude product by column chromatography (4:1 hexanes/ethyl acetate with $\left.0.2 \% \mathrm{NEt}_{3}\right)$ gave $21 \mathrm{mg}(55 \%)$ of $3 \mathrm{c}$ as a clear colorless oil.

Data for 3c: ${ }^{1} \mathrm{HNMR}\left(\mathrm{CDCl}_{3}, 400 \mathrm{MHz}\right) \delta 9.69(\mathrm{~d}, \mathrm{~J}=2.8 \mathrm{~Hz}, 1 \mathrm{H}), 5.61(\mathrm{~s}, 1 \mathrm{H}), 4.30$ (td, $\mathrm{J}=3.6,2 \mathrm{~Hz}, 1 \mathrm{H}$ ), 2.48 (tdd, $\mathrm{J}=10.2,6.6,2.8 \mathrm{~Hz}, 1 \mathrm{H}), 2.30$ (dd, J = 16, $10.2 \mathrm{~Hz}, 1 \mathrm{H}$ ), 2.25$2.19(\mathrm{~m}, 1 \mathrm{H}), 2.15(\mathrm{dd}, \mathrm{J}=16,6.6 \mathrm{~Hz}, 1 \mathrm{H}), 2.06-1.90(\mathrm{~m}, 3 \mathrm{H}), 1.74(\mathrm{~s}, 3 \mathrm{H}), 1.69(\mathrm{dt}, \mathrm{J}=15$, $8.8 \mathrm{~Hz}, 1 \mathrm{H}), 1.36-1.23(\mathrm{~m}, 2 \mathrm{H}) ;{ }^{13} \mathrm{CNMR}\left(\mathrm{CDCl}_{3}, 100 \mathrm{MHz}\right) \delta 203.7,136.6,120.0,71.9,52.6$, 51.1, 39.0, 34.7, 30.5, 26.4, 23.4; FTIR (Thin film) $v$ max 3419 (br), 2963, 2932, 2909, 2717, 1722, 1666, 1435, 1503, 1377, 1346, 1417, 1269, 1154, 1088, 1048, 977, 900, 844, 902, 743, 684, $659 \mathrm{~cm}^{-1}$; HRMS (EI $70 \mathrm{eV}$ ) calcd for $\mathrm{C}_{11} \mathrm{H}_{16} \mathrm{O}_{2} \mathrm{M}^{+} 180.1150 \mathrm{~m} / \mathrm{z}$ found $180.1156 \mathrm{~m} / \mathrm{z}$. Partial reduction of trans-fused methyl ester $\mathbf{3 b}$ with DIBAL gave aldehyde $3 \mathbf{c}$ that was identical to the material obtained from the Lewis-Acid promoted experiment, confirming the stereochemical assignment.

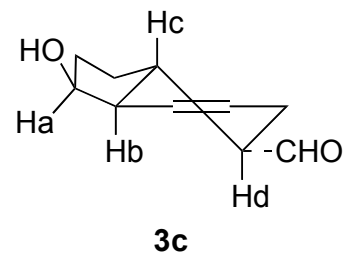

\begin{tabular}{ccc}
\hline Proton & Chemical Shift $(\mathrm{ppm})$ & Multiplicity, $J(\mathrm{~Hz})$ \\
\hline $\mathrm{Ha}$ & 4.29 & td, $3.6,2$ \\
$\mathrm{Hd}$ & 2.48 & tdd $, 10.8,7.6,3.2$
\end{tabular}


Data for 4c (as a 2.5:1 mixture with 3c): ${ }^{1} \mathrm{HNMR}\left(\mathrm{CDCl}_{3}, 400 \mathrm{MHz}\right) \delta 9.69$ (d, J = $2 \mathrm{~Hz}$, 1H), 5.45 (s, 1H), 4.19(td, J = 3.4, $1.4 \mathrm{~Hz}, 1 \mathrm{H}), 2.56$ (tdd, J = 10, 4, $2.4 \mathrm{~Hz}, 1 \mathrm{H}$ ), 2.51 (br s, $1 \mathrm{H}), 2.25(\mathrm{qd}, \mathrm{J}=10.4,4.8 \mathrm{~Hz}, 1 \mathrm{H}), 2.11-2.05(\mathrm{~m}, 2 \mathrm{H}), 1.87-1.82(\mathrm{~m} \mathrm{1H}), 1.81(\mathrm{~s}, 3 \mathrm{H}), 1.80-$ $1.69(\mathrm{~m}, 2 \mathrm{H}), 1.18-1.02(\mathrm{~m}, 1 \mathrm{H}) ;{ }^{13} \mathrm{CNMR}\left(\mathrm{CDCl}_{3}, 100 \mathrm{MHz}\right) \delta ; 204.9,138.0,118.6,74.7$, $46.0,34.0,33.2,28.9,28.2,24.0,14.1$.

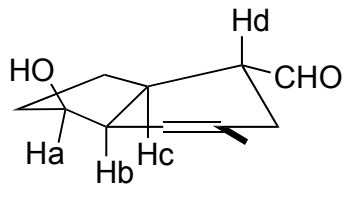

4c

\begin{tabular}{ccc}
\hline Proton & Chemical Shift $(\mathrm{ppm})$ & Multiplicity, $J(\mathrm{~Hz})$ \\
\hline $\mathrm{Ha}$ & 4.19 & td, 3.4, 1.4 \\
$\mathrm{Hb}$ & 2.5 & br s
\end{tabular}

$\mathrm{Hd}$ tdd, 10, 4, 2.4

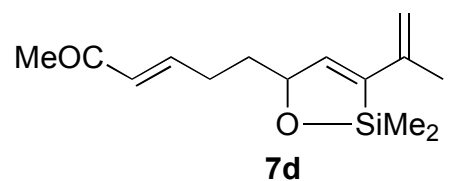

7d
1) $120^{\circ} \mathrm{C}, \mathrm{PhMe}$

2) TBAF, THF $60^{\circ} \mathrm{C}$ $85 \%, 2: 1$ d.r.

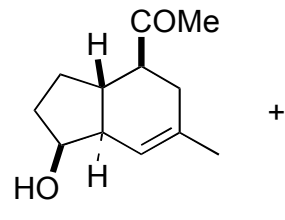

3d

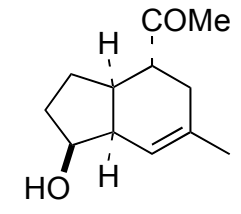

$4 d$

rac-1-((1S,3aS,4S,7aS)-1-Hydroxy-6-methyl-2,3,3a,4,5,7a-hexahydro-1H-inden-4-

yl)-ethanone (3d). Following general procedure $C$, a solution of triene $7 \mathbf{d}(50 \mathrm{mg}, 0.2 \mathrm{mmol}$ ) and 1 crystal of BHT in toluene $(6 \mathrm{~mL})$ was heated to $120^{\circ} \mathrm{C}$ for $20 \mathrm{~h}$, then concentrated. The resulting oil was dissolved in THF $(6 \mathrm{~mL})$ and treated with tetrabutylammonium fluoride trihydrate $(190 \mathrm{mg}, 0.6 \mathrm{mmol})$ at $60{ }^{\circ} \mathrm{C}$ for $1.5 \mathrm{~h}$. Purification of the crude cycloadduct by column chromatography (4:1 hexanes to ethyl acetate) yielded $33 \mathrm{mg} \mathrm{(85 \% )}$ of $\mathbf{3 d}$ and $\mathbf{4 d}$ as an inseparable 2:1 mixture.

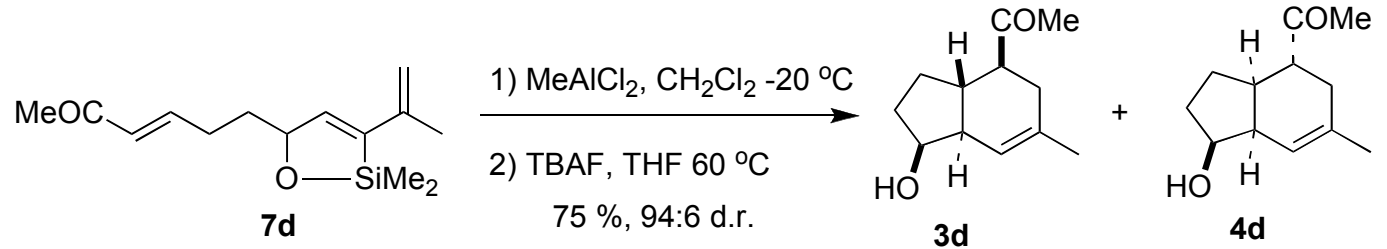

General Procedure D was followed with a solution of triene $7 \mathbf{d}(50 \mathrm{mg}, 0.2 \mathrm{mmol})$ and $\mathrm{MeAICl}_{2}\left(200 \mu \mathrm{L}, 0.2 \mathrm{mmol}, 1 \mathrm{M}\right.$ solution in $\left.\mathrm{CH}_{2} \mathrm{Cl}_{2}\right)$ in $\mathrm{CH}_{2} \mathrm{Cl}_{2}(20 \mathrm{~mL})$ at $-20{ }^{\circ} \mathrm{C}$ for $3.5 \mathrm{~h}$. The crude cycloadduct mixture was then dissolved in THF $(6 \mathrm{~mL})$ and solid tetrabutylammonium fluoride trihydrate $(190 \mathrm{mg}, 0.6 \mathrm{mmol})$ was added. The solution was 
warmed to $60{ }^{\circ} \mathrm{C}$ for $1.5 \mathrm{~h}$. The crude material was purified by column chromatography (3:1 hexanes/ethyl acetate) to yield $29 \mathrm{mg}(75 \%)$ of $\mathbf{3 d}$ as a clear yellow oil.

Data for 3d: ${ }^{1} \mathrm{HNMR}\left(\mathrm{CDCl}_{3}, 400 \mathrm{MHz}\right) \delta 5.58(\mathrm{~s}, 1 \mathrm{H}), 4.20(\mathrm{t}, \mathrm{J}=5.2 \mathrm{~Hz}, 1 \mathrm{H}), 2.61$ (td, $\mathrm{J}=10.4,7.2 \mathrm{~Hz}, 1 \mathrm{H}), 2.25-2.10(\mathrm{~m}, 6 \mathrm{H}), 1.98(\mathrm{qd}, \mathrm{J}=11.4,6.2 \mathrm{~Hz}, 1 \mathrm{H}), 1.91-1.85(\mathrm{~m}, 2 \mathrm{H})$, $1.70(\mathrm{~s}, 3 \mathrm{H}), 1.62$ (dt, J = 15.6, $8.2 \mathrm{~Hz}, 1 \mathrm{H}), 1.14(\mathrm{dq}, 11.2,8 \mathrm{~Hz}, 2 \mathrm{H}) ;{ }^{13} \mathrm{CNMR}\left(\mathrm{CDCl}_{3}, 100\right.$ $\mathrm{MHz}) \delta 211.4,136.9,120.0,72.1,53.5,51.0,40.6,34.6,34.1,28.7,27.0,23.3$; FTIR (Thin film) $v \max 3430$ (br), 2963, 2910, 1702, 1662, 1434, 1355, 1318, 1290, 1261, 1239, 1168, 1154, 1128, 1088, 1033, 1020, 964, 945, 909, 848, 798, $732 \mathrm{~cm}^{-1}$; HRMS (El $\left.70 \mathrm{eV}\right)$ calcd for $\mathrm{C}_{12} \mathrm{H}_{18} \mathrm{O}_{2} \mathrm{M}^{+} 194.1307 \mathrm{~m} / \mathrm{z}$ found $194.1315 \mathrm{~m} / \mathrm{z}$.

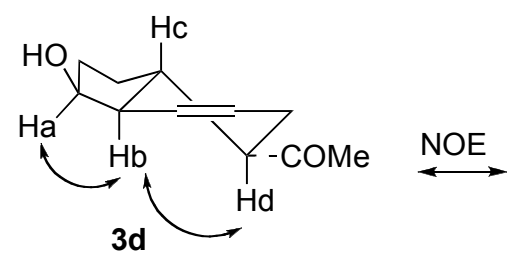

\begin{tabular}{ccc}
\hline Proton & Chemical Shift (ppm) & Multiplicity, $J(\mathrm{~Hz})$ \\
\hline $\mathrm{Ha}$ & 4.23 & $\mathrm{t}, 5.2$ \\
$\mathrm{Hc}$ & 1.98 & $\mathrm{qd} 11.4,6.3$ \\
$\mathrm{Hd}$ & 2.62 & td, $10.4,7.2$
\end{tabular}

Data for 4d (as a 2:1 mixture with 3d): ${ }^{1} \mathrm{HNMR}\left(\mathrm{CDCl}_{3}, 400 \mathrm{MHz}\right) \delta 5.42(\mathrm{~s}, 1 \mathrm{H}), 4.15$ (td, J = 4, $1.2 \mathrm{~Hz}, 1 \mathrm{H}), 2.77$ (dt, J = 16, $4.5 \mathrm{~Hz}, 1 \mathrm{H}$ ) , 2.71 (td, J = 11.2, $4.8 \mathrm{~Hz}, 1 \mathrm{H}$ ), 2.47 (s, $1 \mathrm{H}$ ), $2.32(\mathrm{qd}, \mathrm{J}=8.7,4.9 \mathrm{~Hz}, 1 \mathrm{H}), 2.15(\mathrm{~s}, 3 \mathrm{H}), 2.14-2.12(\mathrm{~m}, 2 \mathrm{H}) 2.11(\mathrm{~d}, \mathrm{~J}=2.32 \mathrm{~Hz}, 1 \mathrm{H})$, $2.06(\mathrm{~d}, \mathrm{~J}=4.8 \mathrm{~Hz}, 1 \mathrm{H}), 1.74(\mathrm{~s}, 3 \mathrm{H}), 1.60-1.56(\mathrm{~m}, 1 \mathrm{H}) ;{ }^{13} \mathrm{CNMR}\left(\mathrm{CDCl}_{3}, 100 \mathrm{MHz}\right) \delta 212.4$, 138.6, 118.5, 75.0, 53.4, 46.6, 36.6, 35.6, 32.8, 29.8, 24.7, 23.9
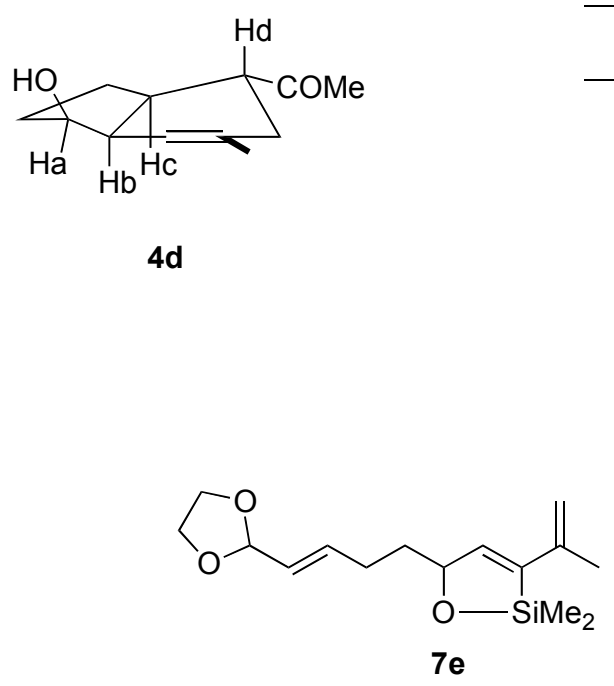

\begin{tabular}{ccc}
\hline Proton & Chemical Shift (ppm) & Multiplicity, $J(\mathrm{~Hz})$ \\
\hline $\mathrm{Ha}$ & 4.16 & $\mathrm{t}, 3.2$ \\
$\mathrm{Hb}$ & 2.46 & $\mathrm{br} \mathrm{s}$ \\
$\mathrm{Hd}$ & 2.71 & $\mathrm{dt}, 11.2,4.8$
\end{tabular}

rac-(1S,3aR,4R,7aS)-4-(1,3-Dioxolan-2-yl)-6-methyl-2,3,3a,4,5,7a-hexahydro-1Hinden-1-ol (4e). Following general procedure $C$ with a solution of triene 7 e $(50 \mathrm{mg}, 0.18$ 
$\mathrm{mmol})$ in toluene $(4 \mathrm{~mL})$ was heated to $160{ }^{\circ} \mathrm{C}$ for $36 \mathrm{~h}$, then concentrated. The crude cycloadduct was dissolved in THF $(3 \mathrm{~mL})$, solid tetrabutylammonium fluoride trihydrate was added $(170 \mathrm{mg}, 0.54 \mathrm{mmol})$ and the mixture was heated to $60{ }^{\circ} \mathrm{C}$ for $1.5 \mathrm{~h}$. The crude cycloadduct was purified by column chromatography ( $4: 1$ hexanes/ethyl acetate) yielding 32 mg $(74 \%)$ of $4 \mathrm{e}$ as a clear colorless oil: ${ }^{1} \mathrm{HNMR}\left(\mathrm{CDCl}_{3}, 400 \mathrm{MHz}\right) \delta 5.39(\mathrm{~s}, 1 \mathrm{H}), 4.86(\mathrm{~d}, \mathrm{~J}=$ $3.2 \mathrm{~Hz}, 1 \mathrm{H}$ ), $4.14(\mathrm{t}, \mathrm{J}=3.7 \mathrm{~Hz}, 1 \mathrm{H}), 3.99-3.80(\mathrm{~m}, 4 \mathrm{H}), 2.49(\mathrm{~s}, 1 \mathrm{H}), 2.10$ (qd, J = 9.6, $5.2 \mathrm{~Hz}$, $1 \mathrm{H}), 2.00(\mathrm{dd}, \mathrm{J}=12.4,8.4 \mathrm{~Hz}, 1 \mathrm{H}), 1.95(\mathrm{dd}, \mathrm{J}=12.4,8.4 \mathrm{~Hz}, 1 \mathrm{H}), 1.85-1.6(\mathrm{~m}, 7 \mathrm{H}), 1.39(\mathrm{~s}$, $1 \mathrm{H}) ;{ }^{13} \mathrm{CNMR}\left(\mathrm{CDCl}_{3}, 100 \mathrm{MHz}\right) \delta 139.4,117.7,105.9,74.9,65.1,64.9,46.4,42.2,35.0,33.2$, 28.4, 28.3, 24.2; FTIR (Thin film) $v \max 3445$ (br), 2957, 2917, 2882, 1717, 1674, 1578, 1541, $1464,1436,1403,1377,1345,1302,1258,1212,1155,1129,1106,1092,1032,992,968$, $945,913,857,803,736,701 \mathrm{~cm}^{-1}$; HRMS (ESI) calcd for $\mathrm{C}_{13} \mathrm{H}_{20} \mathrm{O}_{3}[\mathrm{M}+\mathrm{Na}]^{+} 247.1310 \mathrm{~m} / \mathrm{z}$ found $247.1314 \mathrm{~m} / \mathrm{z}$
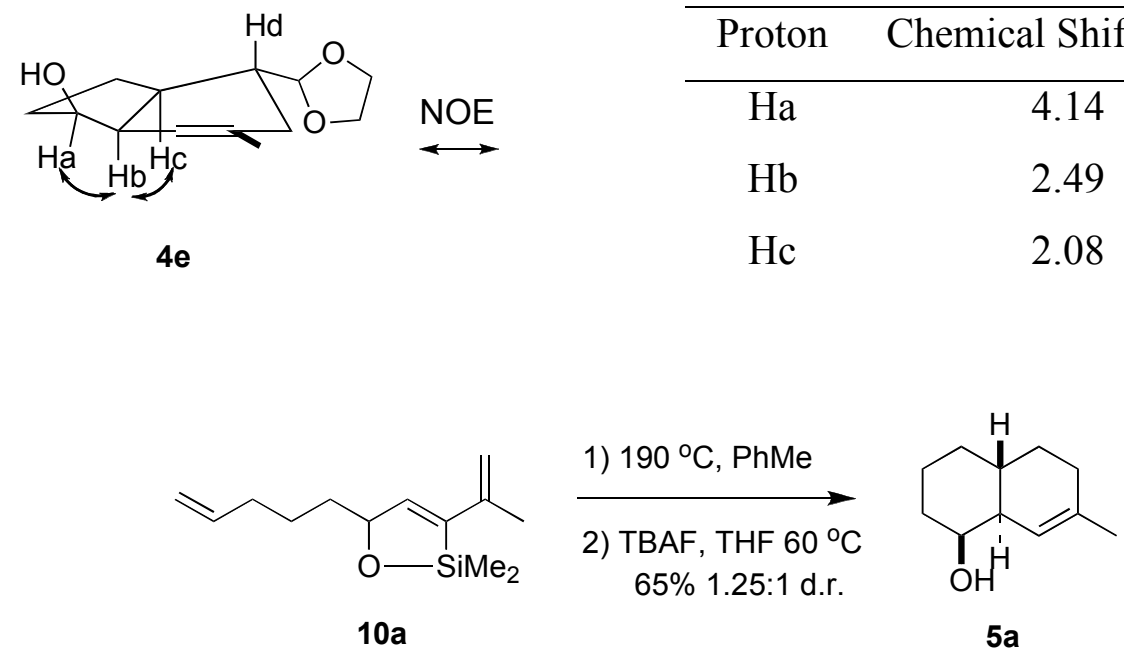

$5 a$

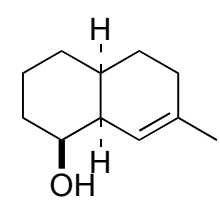

$6 a$

7-Methyl-1,2,3,4,4a,5,6,8a-octahydronaphthalen-1-ol (5a and 6a). Following general procedure $\mathrm{C}$ a solution of triene $10 \mathrm{a}(48.3 \mathrm{mg}, 0.22 \mathrm{mmol})$ and 1 crystal of BHT in toluene $(6 \mathrm{~mL})$ was heated to $190{ }^{\circ} \mathrm{C}$ for $72 \mathrm{~h}$, then concentrated. The crude cycloadduct was dissolved in THF $(3 \mathrm{~mL})$, solid tetrabutylammonium fluoride trihydrate $(210 \mathrm{mg}, 0.66$ mmol) was added and the mixture was heated to $60{ }^{\circ} \mathrm{C}$ for $1.5 \mathrm{~h}$. The crude cycloadduct was purified by column chromatography (6:1 pentane to $\left.\mathrm{Et}_{2} \mathrm{O}\right)$ yielding $12.3 \mathrm{mg} \mathrm{(34 \% )}$ of $\mathbf{5 a}$ as a clear yellow oil and $11.2 \mathrm{mg}$ (31\%) of $6 \mathrm{a}$ as a white crystalline solid.

Data for 5a: ${ }^{1} \mathrm{HNMR}\left(\mathrm{CDCl}_{3}, 400 \mathrm{MHz}\right) \delta 5.19(\mathrm{~s}, 1 \mathrm{H}), 3.92(\mathrm{t}, 2.4 \mathrm{~Hz}, 1 \mathrm{H}) ; 2.11-2.00$ $(\mathrm{m}, 1 \mathrm{H}), 1.90-1.80,(\mathrm{~m}, 2 \mathrm{H}), 1.76-1.61(\mathrm{~m}, 6 \mathrm{H}), 1.60-1.41(\mathrm{~m}, 4 \mathrm{H}), 1.28(\mathrm{qd}, 12,5.6 \mathrm{~Hz}, 1 \mathrm{H})$, 1.01 (qd, J = 12, $4 \mathrm{~Hz}, 1 \mathrm{H}) ;{ }^{13} \mathrm{CNMR}\left(\mathrm{CDCl}_{3}, 100 \mathrm{MHz}\right) \delta 137.9,123.2,69.9,47.0,32.9,32.7$, 
32.6, 30.7, 30.0, 23.7, 20.6; FTIR (Thin film) $v \max 3434,2925,2851,1644,1445,1375$, 1342, 1328, 1290, 1259, 1237, 1200, 1184, 1135, 1115, 1083, 1051, 1001, 981, 964, 922, 899, 860, $826 \mathrm{~cm}^{-1}$; HRMS (El $70 \mathrm{eV}$ ) calcd for $\mathrm{C}_{12} \mathrm{H}_{18} \mathrm{O}_{2} \mathrm{M}^{+} 166.1358 \mathrm{~m} / \mathrm{z}$ found 166.1355 $m / z$.

Data for 6a: ${ }^{1} \mathrm{HNMR}\left(\mathrm{CDCl}_{3}, 400 \mathrm{MHz}\right) \delta 5.33(\mathrm{~s}, 1 \mathrm{H}), 3.69(\mathrm{td}, \mathrm{J}=4.4,2.8 \mathrm{~Hz}, 1 \mathrm{H})$, 2.35 (s, 1H), 2.11-2.01 (ddddd, J = 11.6, 8.8, 5.6, 5.6, 3.6 Hz, 1H), 1.96-1.85 (m, 3H), m 1.83$1.68(\mathrm{~m}, 6 \mathrm{H}), 1.60-1.40(\mathrm{~m}, 4 \mathrm{H}) ;{ }^{13} \mathrm{CNMR}\left(\mathrm{CDCl}_{3}, 100 \mathrm{MHz}\right) \delta 141.2,117.9,75.0,45.8,34.5$, 33.4, 29.5, 29.4, 29.1, 24.3; FTIR (Thin film) $v \max 3350$ (br), 2922, 2883, 2855, 2836, 1435, 1350, 1296, 1271, 1210, 1169, 1142, 1100, 1082, 1051, 1023, 989, 973, 958, 942, 880, 825, $804 \mathrm{~cm}^{-1}$; HRMS (El $\left.70 \mathrm{eV}\right)$ calcd for $\mathrm{C}_{11} \mathrm{H}_{18} \mathrm{O} \mathrm{M} \mathrm{M}^{+} 166.1358 \mathrm{~m} / \mathrm{z}$ found $166.1358 \mathrm{~m} / \mathrm{z}$.

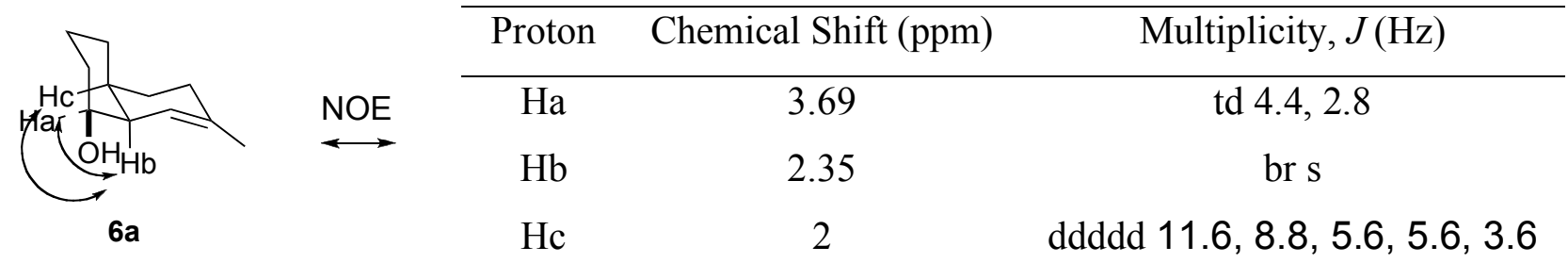

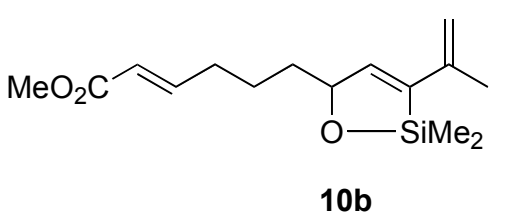

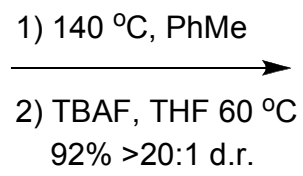
$92 \%>20: 1$ d.r.

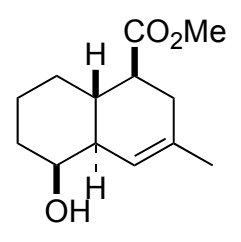

$5 \mathbf{b}$

rac-(1S,4aS,5S,8aS)-Methyl-5-hydroxy-3-methyl-1,2,4a,5,6,7,8,8a-

octahydronaphthalene-1-carboxylate (5b). Following general procedure $\mathrm{C}$, a solution of triene $10 \mathrm{~b}(50 \mathrm{mg}, 0.18 \mathrm{mmol})$, and 1 crystal of $\mathrm{BHT}$ in toluene $(6 \mathrm{~mL})$ was heated to $140{ }^{\circ} \mathrm{C}$ for $20 \mathrm{~h}$, then concentrated. The crude cycloadduct was dissolved in THF (6 mL), solid tetrabutylammonium fluoride trihydrate $(170 \mathrm{mg}, 0.54 \mathrm{mmol})$ was added and the mixture was heated to $60{ }^{\circ} \mathrm{C}$ for $1.5 \mathrm{~h}$. The crude cycloadduct was purified by column chromatography (4:1 hexanes to ethyl acetate) yielding $37 \mathrm{mg} \mathrm{(92 \% )}$ of $\mathbf{5 b}$ as a clear yellow: ${ }^{1} \mathrm{HNMR}\left(\mathrm{CDCl}_{3}\right.$, $400 \mathrm{MHz}) \delta 5.22(\mathrm{~s}, 1 \mathrm{H}), 3.98(\mathrm{~s}, 1 \mathrm{H}), 3.68(\mathrm{~s}, 3 \mathrm{H}), 2.41(\mathrm{dt}, \mathrm{J}=10.8,5.2 \mathrm{~Hz}, 1 \mathrm{H}) 2.36-2.28$ (m, 1H), $2.10(\mathrm{dd}, \mathrm{J}=17.2,4.4 \mathrm{~Hz}, 1 \mathrm{H}), 1.98-1.91(\mathrm{~m}, 1 \mathrm{H}), 1.89-1.82(\mathrm{~m}, 2 \mathrm{H}), 1.66(\mathrm{~s}, 3 \mathrm{H})$, 1.65-1.42 (m, 4H), $1.36(\mathrm{~s}, 1 \mathrm{H}), 1.03(\mathrm{qd}, \mathrm{J}=12.4,3.2 \mathrm{~Hz}, 1 \mathrm{H}) ;{ }^{13} \mathrm{CNMR}\left(\mathrm{CDCl}_{3}, 100 \mathrm{MHz}\right) \delta$ 176.1, 135.7, 69.8, 51.4, 46.5, 46.2, 34.9, 34.0, 32.3, 29.9, 23.3, 20.2; FTIR (Thin film) $v$ max 3446 (br), 2929, 2847, 1374, 1436, 1377, 1340, 13030, 12616, 1194, 1162, 1104, 1081, 1053 , 
1030, 987, 933, 904, 884, 851, 831, 803, 752, $724 \mathrm{~cm}^{-1}$; HRMS (ESI) calcd for $\mathrm{C}_{13} \mathrm{H}_{20} \mathrm{O}_{3}$ $[\mathrm{M}+\mathrm{H}]^{+} 225.1491 \mathrm{~m} / \mathrm{z}$ found $225.1488 \mathrm{~m} / \mathrm{z}$.

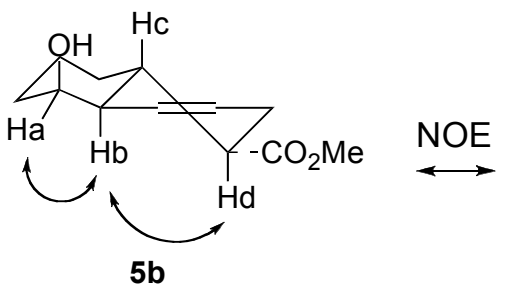

\begin{tabular}{ccc}
\hline Proton & Chemical Shift $(\mathrm{ppm})$ & Multiplicity, $J(\mathrm{~Hz})$ \\
\hline $\mathrm{Ha}$ & 3.97 & $\mathrm{t}, 2.4$ \\
$\mathrm{Hc}$ & 1.85 & $\mathrm{qd} 10.8,2.8$ \\
$\mathrm{Hd}$ & 2.39 & $\mathrm{td}, 10.8,5.2$
\end{tabular}
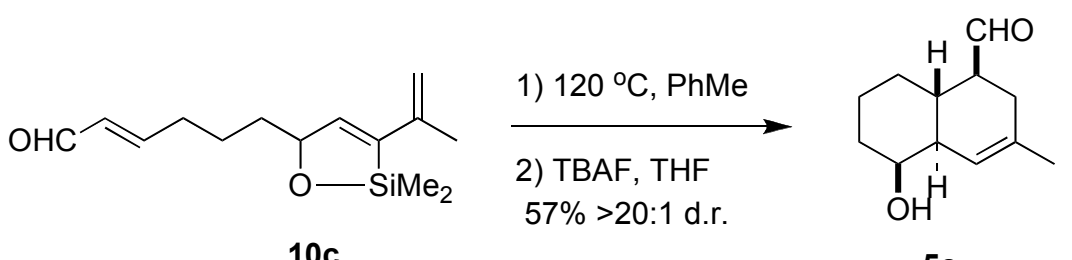

$5 c$

rac-(1S,4aS,5S,8aS)-5-Hydroxy-3-methyl-1,2,4a,5,6,7,8,8a-octahydronaphthalene-

1-carbaldehyde (5c). Following general procedure $C$ a solution of triene $10 \mathrm{c}$ (50 mg, 0.2 $\mathrm{mmol}$ ) and 1 crystal of $\mathrm{BHT}$ in toluene $(6 \mathrm{~mL})$ was heated to $120^{\circ} \mathrm{C}$ for $24 \mathrm{~h}$, then concentrated. The crude cycloadduct was dissolved in THF $(2 \mathrm{~mL})$, solid tetrabutylammonium fluoride trihydrate $(190 \mathrm{mg}, 0.6 \mathrm{mmol})$ was added and the mixture was stirred for $12 \mathrm{~h}$. The crude cycloadduct was purified by column chromatography $(4: 1$ hexanes/ethyl acetate with $0.2 \% \mathrm{NEt}_{3}$ ) yielding $22 \mathrm{mg} \mathrm{(57 \% )}$ of $5 \mathrm{c}$ as a clear yellow oil.

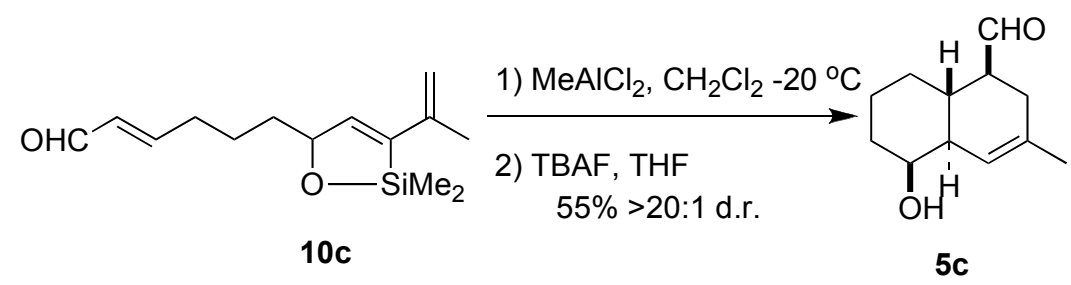

Following general procedure $\mathrm{D}$, a solution of triene $10 \mathrm{c}$ (50 $\mathrm{mg}, 0.2 \mathrm{mmol})$ in $\mathrm{CH}_{2} \mathrm{Cl}_{2}$ $(20 \mathrm{~mL})$ was treated with $\mathrm{MeAlCl}_{2}(200 \mu \mathrm{L}, 0.2 \mathrm{mmol})$ for $6 \mathrm{~h}$ at $-20^{\circ} \mathrm{C}$. The crude cycloadduct was then dissolved in THF $(2 \mathrm{~mL})$ and tetrabutylammonium fluoride trihydrate (190 $\mathrm{mg}, 0.6 \mathrm{mmol}$ ) and stirred for $12 \mathrm{~h}$ at room temperature. The crude material was purified by column chromatography ( $4: 1$ hexanes:ethyl acetate with $0.2 \% \mathrm{NEt}_{3}$ ) to yield $21 \mathrm{mg}$ (55\%) of 5c as a clear yellow oil: ${ }^{1} \mathrm{HNMR}\left(\mathrm{CDCl}_{3}, 400 \mathrm{MHz}\right) \delta 9.59(\mathrm{~d}, \mathrm{~J}=4.4 \mathrm{~Hz}, 1 \mathrm{H}), 5.20(\mathrm{~s}$, $1 \mathrm{H}), 4.14(\mathrm{~d}, \mathrm{~J}=2 \mathrm{~Hz}, 1 \mathrm{H}), 2.31$ (tdd, $\mathrm{J}=10,5.6,4.4 \mathrm{~Hz}, 1 \mathrm{H}), 2.18(\mathrm{dd}, \mathrm{J}=\mathrm{XXXX} \mathrm{Hz,1H}$ ), 
2.03-1.85 (m, 4H), 1.80-1.72 (m, 3H), $1.71(\mathrm{~s}, 3 \mathrm{H}), 1.55-1.43(\mathrm{~m}, 2 \mathrm{H}), 1.06(\mathrm{qd}, \mathrm{J}=13.6$, $4.4 \mathrm{~Hz}, 1 \mathrm{H}) ;{ }^{13} \mathrm{CNMR}\left(\mathrm{CDCl}_{3}, 100 \mathrm{MHz}\right) \delta 205.1,132.1,125.2,70.4,52.7,45.9,33.8,32.6$, 30.2, 29.5, 23.1, 20.1; FTIR (Thin film) $v \max 3401$ (br), 2957, 2918, 2850, 2709, 1724, 1682 , $1578,1541,1444,1405,1377,1338,1255,1215,1196,1147,1130,1109,1088,1071,1026$, 947, 934, 885, 830, 788, $678 \mathrm{~cm}^{-1}$; HRMS (El $70 \mathrm{eV}$ ) calcd for $\mathrm{C}_{12} \mathrm{H}_{18} \mathrm{O}_{2} \mathrm{M}^{+} 194.1307 \mathrm{~m} / \mathrm{z}$ found $194.1314 \mathrm{~m} / \mathrm{z}$.

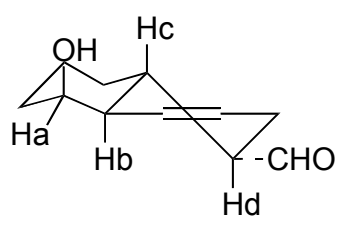

$5 c$

\begin{tabular}{ccc}
\hline Proton & Chemical Shift $(\mathrm{ppm})$ & Multiplicity, $J(\mathrm{~Hz})$ \\
\hline $\mathrm{Ha}$ & 4.01 & $\mathrm{t} 1.6$ \\
$\mathrm{Hb}$ & 1.92 & $\mathrm{dd} 10.8,2.8$ \\
$\mathrm{Hd}$ & 2.31 & $\mathrm{tdd}, 10,5.6,4.4$
\end{tabular}

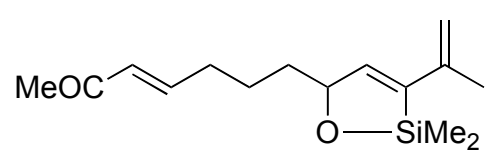

$10 \mathrm{~d}$
1) $120^{\circ} \mathrm{C}, \mathrm{PhMe}$

2) TBAF, THF $60^{\circ} \mathrm{C}$ $93 \% 97: 3$ d.r.

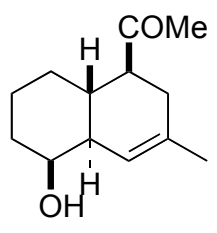

5d

rac-(1S,4aS,5S,8aS)-Methyl-5-hydroxy-3-methyl-1,2,4a,5,6,7,8,8a-

octahydronaphthalene-1-carboxylate (5d). Following general procedure $C$, a solution of triene $10 \mathrm{~d}(50 \mathrm{mg}, 0.19 \mathrm{mmol})$, and 1 crystal of $\mathrm{BHT}$ in toluene $(6 \mathrm{~mL})$ was heated to $120{ }^{\circ} \mathrm{C}$ for $20 \mathrm{~h}$, then concentrated. The crude cycloadduct was dissolved in THF (6 mL), solid tetrabutylammonium fluoride trihydrate $(180 \mathrm{mg}, 0.57 \mathrm{mmol})$ was added and the mixture was stirred for $1.5 \mathrm{~h}$ at $60{ }^{\circ} \mathrm{C}$. Purification of the crude product by column chromatography $(4: 1$ hexanes/ethyl acetate) yielded $37.5 \mathrm{mg}$ (93\%) of $\mathbf{5 d}$ as a clear yellow.

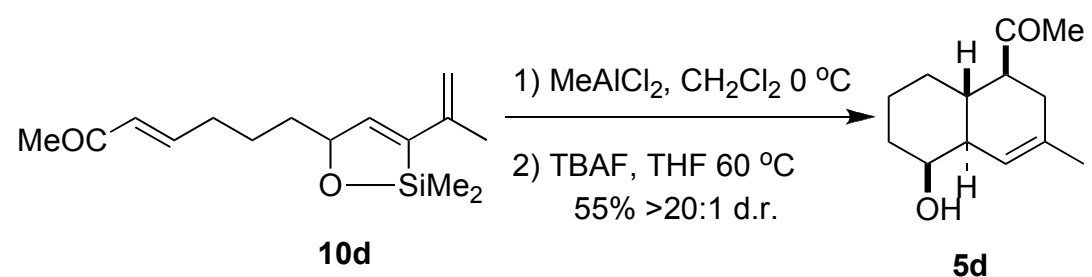

Following general procedure $\mathrm{D}$, a solution of triene $10 \mathrm{~d}(50 \mathrm{mg}, 0.19 \mathrm{mmol})$ in $\mathrm{CH}_{2} \mathrm{Cl}_{2}$ $(19 \mathrm{~mL})$ was treated with $\mathrm{MeAICl}_{2}\left(190 \mu \mathrm{L}, 0.19 \mathrm{mmol}, 1 \mathrm{M}\right.$ in $\left.\mathrm{CH}_{2} \mathrm{Cl}_{2}\right)$ for $6 \mathrm{~h}$ at $0{ }^{\circ} \mathrm{C}$. The crude cycloadduct was then dissolved in THF $(3 \mathrm{~mL})$ and treated with tetrabutylammonium fluoride trihydrate $(110 \mathrm{mg}, 0.35 \mathrm{mmol})$ was for $1.5 \mathrm{~h}$ at $60{ }^{\circ} \mathrm{C}$. The crude material was 
purified by column chromatography ( $4: 1$ hexanes/ethyl acetate) to yield $13.5 \mathrm{mg}(57 \%)$ of $\mathbf{5 d}$ as a clear yellow: ${ }^{1} \mathrm{HNMR}\left(\mathrm{CDCl}_{3}, 400 \mathrm{MHz}\right) \delta 5.22(\mathrm{~s}, 1 \mathrm{H}), 3.98(\mathrm{t}, \mathrm{J}=2 \mathrm{~Hz}, 1 \mathrm{H}), 2.54(\mathrm{td}, \mathrm{J}=$ 13.9, $5.6 \mathrm{~Hz}, 1 \mathrm{H}), 2.20-2.11(\mathrm{~m}, 4 \mathrm{H}), 2.00(\mathrm{dd}, \mathrm{J}=17.6 \mathrm{~Hz}, 5.2 \mathrm{~Hz}, 1 \mathrm{H}), 1.95-1.83(\mathrm{~m}, 3 \mathrm{H})$, $1.72-1.38(\mathrm{~m} \mathrm{8H}), 0.99$ (qd, J = 12.4, $3.2 \mathrm{~Hz}, 1 \mathrm{H}) ;{ }^{13} \mathrm{CNMR}\left(\mathrm{CDCl}_{3}, 100 \mathrm{MHz}\right) \delta 212.7,135.5$, 123.4, 69.8, 54.0, 46.3, 34.7, 33.5, 32.3, 29.9, 29.4, 23.3, 20.3; FTIR (Thin film) $v$ max 3436 (br), 2926, 2849, 1702, 1433, 1354, 1257, 1236, 1213, 1165, 1142, 1105, 1081, 1064, 1050, 1002, 976, 904, 882, 846, 831, $803 \mathrm{~cm}^{-1}$; HRMS (ESI) calcd for $\mathrm{C}_{13} \mathrm{H}_{20} \mathrm{O}_{2}[\mathrm{M}+\mathrm{Na}]^{+} 231.1361$ $\mathrm{m} / \mathrm{z}$ found $231.1351 \mathrm{~m} / \mathrm{z}$.

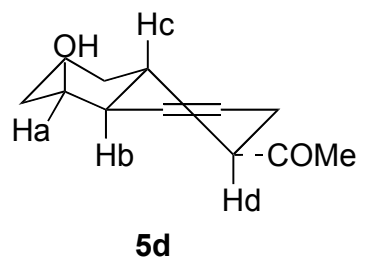

5d

\begin{tabular}{ccc}
\hline Proton & Chemical Shift $(\mathrm{ppm})$ & Multiplicity, $J(\mathrm{~Hz})$ \\
\hline $\mathrm{Ha}$ & 3.98 & $\mathrm{t} 2$ \\
$\mathrm{Hc}$ & 1.86 & $\mathrm{qd} \mathrm{11.2,2.8}$ \\
$\mathrm{Hd}$ & 2.54 & $\mathrm{td}, 11.2,5.6$
\end{tabular}

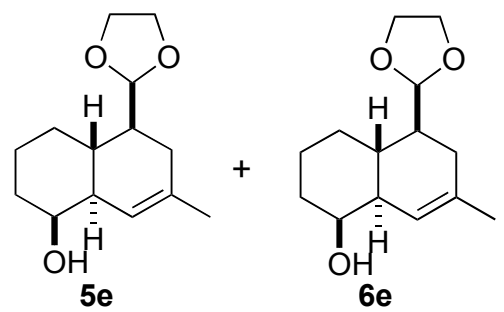

rac-(1S,4aS,5S,8aS)-5-(1,3-Dioxolan-2-yl)-7-methyl-1,2,3,4,4a,5,6,8a-

octahydronaphthalen-1-ol (5e and $6 \mathrm{e}$ ). Following general procedure $C$, a solution of $\mathbf{1 0 d}$ (50 mg, $0.17 \mathrm{mmol}$ ) and 1 crystal of BHT in toluene $\left(6 \mathrm{~mL}\right.$ ) was heated to $190{ }^{\circ} \mathrm{C}$ for $72 \mathrm{~h}$, then concentrated. The crude mixture was dissolved in THF $(6 \mathrm{~mL})$ and treated with solid tetrabutylammonium fluoride trihydrate $(160 \mathrm{mg}, 0.51 \mathrm{mmol})$ for $1.5 \mathrm{~h}$ at $60{ }^{\circ} \mathrm{C}$. Purification of the crude product by column chromatography (6:1 hexanes/ethyl acetate) yielded $14 \mathrm{mg}$ (35 $\%)$ of $5 \mathbf{e}$ as a clear yellow oil and $9 \mathrm{mg}(22 \%)$ of $\mathbf{6 e}$ as a clear yellow oil.

Data for 5e: ${ }^{1} \mathrm{HNMR}\left(\mathrm{CDCl}_{3}, 400 \mathrm{MHz}\right) \delta 5.17(\mathrm{~s}, 1 \mathrm{H}), 4.98(\mathrm{~d}, \mathrm{~J}=3.6 \mathrm{~Hz}, 1 \mathrm{H}), 4.00-$ $3.83(\mathrm{~m}, 5 \mathrm{H}), 2.04-1.95(\mathrm{~m}, 4 \mathrm{H}), 1.88-1.71(\mathrm{~m}, 2 \mathrm{H}), 1.69(\mathrm{~s}, 3 \mathrm{H}), 1.64(\mathrm{dd}, \mathrm{J}=11.2,2.4 \mathrm{~Hz}$, $1 \mathrm{H}), 1.60-1.52(\mathrm{~m}, 1 \mathrm{H}), 1.44(\mathrm{tt}, \mathrm{J}=13.6,2.8 \mathrm{~Hz}, 2 \mathrm{H}), 1.02(\mathrm{qd}, \mathrm{J}=12.4,3.6 \mathrm{~Hz}, 1 \mathrm{H})$; ${ }^{13} \mathrm{CNMR}\left(\mathrm{CDCl}_{3}, 100 \mathrm{MHz}\right) \delta 137.1,122.8,104.6,69.9,65.1,64.9,47.1,41.8,34.3,32.1$, 29.7, 29.0, 23.5, 20.3; FTIR (Thin film) $v$ max 3459 (br), 2927, 2884, 2681, 2236, 1714, 1677, $1640,1444,1401,1376,1329,1299,1258,1212,1189,1150,1101,1081$, 1054, 1035, 999, 
971, 946, 893, 835, $732 \mathrm{~cm}-1$; HRMS (ESI) calcd for $\mathrm{C}_{14} \mathrm{H}_{22} \mathrm{O}_{3}[\mathrm{M}+\mathrm{Na}]^{+} 261.1467 \mathrm{~m} / \mathrm{z}$ found $261.1456 \mathrm{~m} / \mathrm{z}$.

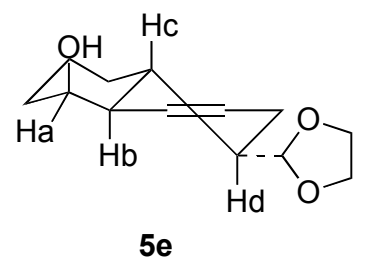

\begin{tabular}{ccc}
\hline Proton & Chemical Shift $(\mathrm{ppm})$ & Multiplicity, $J(\mathrm{~Hz})$ \\
\hline $\mathrm{Hb}$ & 1.86 & $\mathrm{dd} 10.8,2.8$ \\
$\mathrm{Hc}$ & 1.80 & qd $10.8,2.8$
\end{tabular}

Data for 6e: ${ }^{1} \mathrm{HNMR}\left(\mathrm{CDCl}_{3}, 400 \mathrm{MHz}\right) \delta 5.47(\mathrm{~s}, 1 \mathrm{H}), 4.78(\mathrm{~d}, \mathrm{~J}=7.2 \mathrm{~Hz}, 1 \mathrm{H}), 4.00-$ $3.80(\mathrm{~m}, 4 \mathrm{H}), 3.75(\mathrm{dt}, \mathrm{J}=8.8,4 \mathrm{~Hz}, 1 \mathrm{H}), 2.56(\mathrm{~s}, 1 \mathrm{H}), 2.12(\mathrm{~m}, 1 \mathrm{H}), 2.01(\mathrm{~s}, 1 \mathrm{H}), 1.98-1.85$ $(\mathrm{m}, 2 \mathrm{H}), 1.53-1.66(\mathrm{~m}, 4 \mathrm{H}), 1.65-1.56(\mathrm{~m}, 2 \mathrm{H}), 1.47-1.21(\mathrm{~m}, 5 \mathrm{H}) ;{ }^{13} \mathrm{CNMR}\left(\mathrm{CDCl}_{3}, 100 \mathrm{MHz}\right)$ $\delta$ 137.1, 122.8, 104.6, 69.9, 65.1, 64.9, 47.1, 41.8, 34.3, 32.1, 29.7, 29.0, 23.5, 20.3; FTIR (Thin film) $v \max 3401,2929,2856,1446,1404,1374,1352,1326,1303,1270,1212,1131$, 1097, 1072, 1054, 1024, 978, 943, 910, 897, 827, $743 \mathrm{~cm}-1$; HRMS (ESI) calcd for $\mathrm{C}_{14} \mathrm{H}_{22} \mathrm{O}_{3}$ $[\mathrm{M}+\mathrm{Na}]^{+} 261.1467 \mathrm{~m} / \mathrm{z}$ found $261.1465 \mathrm{~m} / \mathrm{z}$.

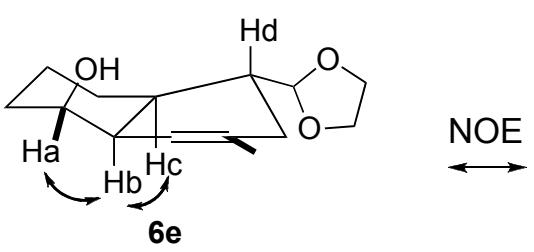

\begin{tabular}{ccc}
\hline Proton & Chemical Shift (ppm) & Multiplicity, $J(\mathrm{~Hz})$ \\
\hline $\mathrm{Ha}$ & 3.75 & $\mathrm{dt} 8.8,4$ \\
$\mathrm{Hb}$ & 2.56 & br s \\
$\mathrm{Hc}$ & 1.92 & dddd $10,8.8,5.6,4$
\end{tabular}

